\title{
1 Drug-driven reclassification of multiple tumour subtypes reveals intrinsic \\ 2 molecular concordance of therapy across histologically disparate cancers
}

3 Yue $\mathrm{Xu}^{1, \#}$, Jie Zheng ${ }^{1, \#}$, Zhaoqing Cai ${ }^{1}$, Wang $\mathrm{Li}^{2}$, Jens Köhler ${ }^{3}$, Yao Dai ${ }^{1}$, Xiaojie Cheng ${ }^{1}$, Tao

$4 \quad \mathrm{Wu}^{1}$, Fan Zhang ${ }^{2,1, *}$, Haiyun Wang ${ }^{1, *}$

5

$6 \quad{ }^{1}$ School of Life Sciences and Technology, Tongji University, Shanghai 200092, China

$7 \quad{ }^{2}$ Department of Thoracic Surgery, Shanghai Pulmonary Hospital, Tongji University School of

8 Medicine, 507 Zhengmin Road, Shanghai 200433, China

$9{ }^{3}$ Department of Medical Oncology, Dana-Farber Cancer Institute, Boston, MA 02215, USA

$11{ }^{*}$ Corresponding authors

12 "Co-first authors

$14{ }^{*}$ Correspondence to: Haiyun Wang, School of Life Sciences and Technology, Tongji University,

15 Shanghai 200092, China; Fan Zhang, Department of Thoracic Surgery, Shanghai Pulmonary

16 Hospital, Tongji University School of Medicine, 507 Zhengmin Road, Shanghai 200433, China.

17

18 Running title: Reclassifying tumors from intrinsic therapeutic concordance

19

20 Keywords: Tumour classification, pharmacogenomics data, pharmacological subtypes, precision

21 medicine, drug sensitivity 
bioRxiv preprint doi: https://doi.org/10.1101/2021.03.02.433516; this version posted March 2, 2021. The copyright holder for this preprint (which was not certified by peer review) is the author/funder. All rights reserved. No reuse allowed without permission.

\section{Email addresses}

24 YX: yue.xu@tongji.edu.cn

25 JZ: 1931524@tongji.edu.cn

26 ZC: 1731473@tongji.edu.cn

27 WL: liwang19902010@163.com

28 JK: jens_kohler@dfci.harvard.edu

29 YD: daiyao0808@sina.com

30 XC: siyecaodelvlanzi@163.com

31 TW: 15708915219@163.com

32 FZ: fzhang@tongji.edu.cn

33 HW: wanghaiyun@tongji.edu.cn 


\section{Abstract}

35 Cancers that are histologically defined as the same type of cancer often need a distinct therapy

36 based on underlying heterogeneity; likewise, histologically disparate cancers can require similar

37 treatment approaches due to intrinsic similarities. A comprehensive analysis integrated with drug

38 response data and genomic alterations, particularly to reveal therapeutic concordance

39 mechanisms across histologically disparate tumour subtypes, has not yet been fully exploited.

40 In this study, we used pharmacogenomic profiling data provided from the Cancer Genome Project

41 (CGP) in a systematic in silico investigation of the pharmacological subtypes of cancers and the

42 intrinsic concordance of molecular mechanisms leading to similar therapeutic responses across

43 histologically disparate tumour subtypes. We further developed a novel approach to redefine cell-

44 to-cell similarity and drug-to-drug similarity from the therapeutic concordance, providing a new

45 point of view to study cancer heterogeneity.

46 Our study identified that histologically different tumours, such as malignant melanoma and 47 colorectal adenocarcinoma, could belong to the same pharmacological subtype regarding drug

48 sensitivity to MEK inhibitors, which was determined by their genomic alterations, high occurrence

49 of BRAF or KRAS mutations. Therapeutic concordance for chemotherapy drugs was identified 50 across histologically disparate hematological tumors mainly due to the extraordinary activation of

51 the cell cycle in blood cancers. A subcluster of SCLC had a more similar profile with

52 hematological tumors, and was associated with the malignant phenotype, with a higher level of

53 MYC expression. We developed a website to store and visualize the pharmacological subtypes of

54 drugs, as well as their connected genomic and expression alterations. 


\section{Introduction}

56 Traditional tumour classification based on histopathologic diagnosis and the TNM staging system

57 offers highly practical guidance for surgical resection, regional radiotherapy or chemotherapies.

58 However, due to complex intertumour and intratumour heterogeneity, cancer patients with such

59 histological diagnoses often suffer from receiving effective drug treatment and therapeutic

60 resistance [1-3]. One of the key mechanisms behind this significant challenge is the fact that

61 cancers from the same tissue of origin often present quite different mechanisms of oncogenesis

62 at the molecular level $[2,3]$. Decades of studies have focused on finding molecular subtypes

63 within histopathologically defined tumour types by analysing large-scale genomic, transcriptomic,

64 proteomic and epigenomic alterations [4]. As a result, a variety of molecular signatures have been

65 identified to distinguish intrinsic molecular subtypes associated with patient survival, prognosis

66 and response to different therapeutic modalities. For example, BRAF mutation in melanoma [5];

67 EGFR-mutant lung adenocarcinomas [6]; luminal A, luminal B, HER2-enriched, basal-like and

68 normal-like subtypes in breast cancer [7-9]; and four prominent genetic subtypes in Diffuse large

69 B-cell lymphoma (DLBCL), termed MCD based on the co-occurrence of MYD88L265P and

70 CD79B mutations, BN2 based on BCL6 fusions and NOTCH2 mutations, N1 based on NOTCH1

71 mutations, and EZB based on EZH2 mutations and BCL2 translocations [10] are several well-

72 established molecular subtypes with explicit clinical significance.

74 With these advances in challenging cancer heterogeneity by identifying subtypes within the same 75 tissues, emerging studies are uncovering facts in the other direction that cancers across

76 disparate tissues of origin can explicitly share common molecular mechanism of oncogenesis [11,

77 12]. For example, one study based on a large-scale genomic analysis revealed that lung

78 squamous, head and neck, and a subset of bladder cancers shared highly concordant signatures

79 typified by TP53 alterations, TP63 amplifications, and high expression of immune and

80 proliferation pathway genes, implicating that those different cancers perhaps require similar

81 treatment approaches [12]. Another study based on large-scale genomic data found that TP53 
82 and KRAS were mutually exclusive in COAD, READ, and LUAD, but significantly coexisted in

83 PAAD. These observations reveal the feasibility of considering the same treatment strategy in

84 different tumor types [13]. Similarly, HER2-targeted therapy may be applied to other cancer types

85 analogous to breast cancer because ERBB2/HER2, which can be amplified in breast cancer, is

86 also mutated and/or amplified in subsets of glioblastoma and gastric, serous endometrial, bladder

87 and lung cancers [11]. In recent years, the FDA granted approval to larotrectinib, which had

88 marked and durable antitumour activity in a variety of patients with RTK fusion-positive cancer,

89 regardless of age or tumour tissue type [13]. Such examples explicitly illuminate a distinct avenue

90 for reclassifying multiple tumour types independent of histopathologic diagnosis, highlighting that

91 treatment approaches specifically discovered in one disease can be applied to another due to

92 their intrinsic concordance of molecular patterns.

94 Currently, emerging efforts, such as pan-cancer analysis projects, are being conducted to

95 comprehensively define commonalities and differences across cancer types and tissues of origin

96 [11]. Nonetheless, integrative analysis, particularly towards revealing intrinsic therapeutic

97 concordance across histologically disparate tumour subtypes, has not yet been fully exploited.

98 Currently, a large-scale pharmacogenomics study, the Cancer Genome Project (CGP), provides

99 high-throughput genomic information and pharmacological profiling of anticancer drugs across

100 hundreds of cell lines that represent explicit molecular subtypes of histologically defined tumours

101 [14]. Hence, we integrated drug response information to pharmacological reclassify tumour

102 subtypes regardless of their tissues of origin. Tumour cells in the same class present similar drug

103 responses, and those in different classes show varied drug responses. Furthermore, by

104 integrating genomic alteration and expression information, we unravelled the intrinsic concordant

105 molecular mechanism associated with the common drug response across histologically disparate

106 cancers. Importantly, this research provides us with a purely therapy-oriented perspective to re-

107 examine tumour classifications independent of histology subtypes. 


\section{Material and Methods}

110 Data from a large-scale pharmacogenomics study, the Cancer Genome Project (CGP), was

111 accessible from its website: http://www.cancerrxgene.org. Gene expression, mutation and drug

112 sensitivity data were downloaded. The CGP dataset includes 987 cell lines, genome-wide

113 analysis of mutations, copy number variations and expression profiling, as well as the presence of

114 commonly rearranged cancer genes, and 367 pharmacological profiles (dataset version 2020)

115 [14]. In these data, the natural logarithm of the IC50 value represents the drug sensitivity value.

116 IC50 is the half maximal inhibitory concentration of an anticancer drug, and a lower value means

117 more sensitivity. Cell lines cannot be classified according to histological subtypes provided by

118 TCGA, labelled as "UNCLASSIFIED", were excluded from our study.

\section{Generating the pharmacological subtypes tree}

121 The cell lines, IC50 of which are smaller than maximal tested concentrations, are defined as 122 sensitive, otherwise resistant. Our method iteratively splits the cancer cells into two groups in a 123 way that gains the best separation of drug sensitivity between two groups until reaching two 124 terminal conditions: The $p$ value of the difference of two groups' drug sensitivity values is smaller 125 than 0.05 or all cancer cells in a node are sensitive or resistant.

127 Suppose the set of all cells is $S_{\text {total }}$, and each cell has its drug sensitivity value. The procedure to 128 grow a pharmacological subtype tree is as follows:

129 (1) Define the root node. Set $S$ as the set of cancer cells in this node, and assign $S_{\text {total }}$ to $S$.

130 (2) Define the root node as the current node.

131 (3) $N$ denotes the number of cells in the current node. If $N$ is smaller than 6 or all cells in such 132 node are sensitive or resistant, finish the procedure of growing the tree. Otherwise, a heuristic 
133 algorithm splits $S$ into two child nodes, the left node and the right node, which achieves the 134 greatest drug sensitivity difference.

135 (4) If no way can be found to split the current node $(p<0.05)$, finish the procedure of growing the 136 tree. Otherwise, the current node is split into two child nodes.

137 (5) Define the left node and right node. Suppose the set of cells in the left node is $S_{l}$ and the set 138 in the right node is $S_{r} . S_{l}$ and $S_{r}$ are the subsets of $S$.

139 (6) Define the left node as the current node. Set $S$ as the set of cells in this node, and assign $S_{l}$ to

$140 \quad$ S. Repeat steps 3-5.

141 (7) Define the right node as the current node. Set $S$ as the set of cells in this node, and assign $142 S_{r}$ to $S$. Repeat steps 3-5.

\section{Algorithm for splitting the node}

145 We used a heuristic algorithm to search for a reasonable number of divisions to split $S$ into $S_{l}$ and

$146 S_{r}$. Suppose the number of the cells set is $n s$. We first sorted the cells by IC50 ascending and

147 divided the cells with higher drug sensitivity into the $S_{l}$ group and the cells with lower drug

148 sensitivity into the $S_{r}$ group. Since each node at least 6 cells, a total of $n s-5$ divisions were 149 considered.

151 For each division, the Mann-Whitney $U$ test was applied to calculate the $p$ value of drug

152 sensitivity between $S_{l}$ and $S_{r}$. The division with the smallest $p$ value was selected as the 153 optimized one to split the cancer cells in the current node. 
156 The similarity of cells is calculated based on their response to the different drugs. For each drug

$157 k$, a pharmacological subtypes tree $S^{k}$ is generated, consisting of $n$ subtypes:

$158 S^{k}=\left(S_{1}^{k}, S_{2}^{k}, \ldots, S_{n}^{k}\right)=\left(S_{t}^{k}\right)_{t=1 \rightarrow n}$

159 Based on this tree, a matrix that defines a similarity of cancer cells, regarding whether they are in

160 the same subtype and their drug response to drug $k$, is calculated. We assume there are $m$ cell

161 lines tested for drug $k$. The cell lines are labelled as sensitive, resistant, or other ones. A sensitive

162 cell line has its IC50 value smaller than minimum tested concentration; a resistant cell line has its

163 IC50 value greater than maximal tested concentration. For two given cell lines $i$ and $j$, their

164 similarity matrix $\left(D_{i j}^{k}\right)$ is defined as:

$$
D_{i j}^{k}=\left\{\begin{array}{c}
1, \quad \text { if }(i, j) \in S_{t}^{k} \text { and both are labelled as sensitive ones } \\
-1, \quad \text { if }(i, j) \in S_{t}^{k} \text { and both are labelled as resistant ones } \\
0, \quad \text { otherwise }
\end{array}\right.
$$

165 If two cell lines $i$ and $j$ are in the same subtype and both are sensitive to drug $k$, their score is 1 ,

166 and -1 for both cell lines with a resistant response, while if they are not in the same subtype or

167 show opposite response to the drug, their score is 0 . A $m \times m$ similarity matrix of cell lines is then

168 generated.

169

170 Then, the similarity of cell lines $i$ and $j$ can be further calculated as:

$171 \quad$ Cell $_{\text {sim }}=\frac{\sum_{k=1}^{q}\left|D_{i j}^{k}\right|}{q}, i \neq j$

172 Wherein $i$ and $j$ denote the cell lines tested for $q$ drugs.

173

174 Furthermore, for two given drugs $a$ and $b$, their similarity is calculated as the summarized

175 similarity across the same cell lines which are tested for both drugs.

$176 \quad \operatorname{Drug}_{\operatorname{sim}}=\frac{\sum_{i=1}^{m} \sum_{j=1}^{m} D_{i j}^{a} \cdot D_{i j}^{b}}{\sum_{i=1}^{m} \Sigma_{j=1}^{m} D_{i j}^{a} \cdot D_{i j}^{a}+\sum_{i=1}^{m} \sum_{j=1}^{m} D_{i j}^{b} \cdot D_{i j}^{b}-\sum_{i=1}^{m} \Sigma_{j=1}^{m} D_{i j}^{a} \cdot D_{i j}^{b}}, \quad i \neq j$ 
177 Wherein $i$ and $j$ denote the cell lines tested for both drugs, and $m$ is the total number of the same 178 cell lines.

180 Connecting pharmacological subtypes with genomic alterations

181 The chi-squared test was used to calculate the connection between genomic alterations,

182 including mutation and translocation, and pharmacological subtypes. Genomic alterations were

183 determined with $p$ values corrected with the Benjamini-Hochberg method for controlling the false

184 discovery rate [15]. Here we used the corrected $p$ value $<0.05$ as the threshold. We defined two

185 types of connections: positive and negative. If pharmacological subtypes with high drug sensitivity

186 had more frequent alteration occurrences, we defined this connection as positive. If the opposite

187 was true, we defined the connection as negative.

189 The connection between mRNA expression and pharmacological subtypes was determined with $p$

190 values from the Kruskal-Wallis $\mathrm{H}$ test corrected with the Benjamini-Hochberg method. If

191 pharmacological subtypes with high drug sensitivity had higher expression, we defined this

192 connection as positive. If the opposite was true, we defined the connection as negative. Here we

193 used the corrected $p$ value $<0.05$ as the threshold. The individual subtypes with sensitive and

194 resistant cells mixed were excluded in the above analyses.

\section{Gene Set Enrichment Analysis (GSEA)}

197 Gene set enrichment analysis (GSEA) [16] was employed to determine the 333 gene sets from

198 KEGG, enriched by a pre-ranked list of all genes, which were sorted by the statistical significance

199 of differential expression defined by DESeq2 analysis [17]. Gene sets with FDR $<0.05$ were 200 statistically significant. 


\section{Statistical analysis}

203 The Fisher's exact test was used to respectively determine whether there is a significant 204 association between pathways and clusters of drugs determined by the number of drug 205 pharmacological subtypes across 6 cancer types or not, whether any difference of phenotypes 206 (cancers/pathways) in clusters of cell lines/drugs derived from the similarity matrix was significant, 207 and whether cancer censor genes are enriched in a list of genes with the most connections to

208 pharmacological subtypes. A hypothesis test based on hypergeometric distribution was used to 209 determine whether a histological cancer is enriched in the most sensitive pharmacological 210 subtype of a drug. The $p$ value $<0.05$ was regarded as statistical significant. Kolmogorov-

211 Smirnov test (K-S test) was employed to compare cumulative distribution function (CDF) of the 212 number of pharmacological subtypes across the drugs between two histological cancers.

\section{Results}

215 We analysed 367 drugs in the CGP dataset and established their pharmacological subtype trees.

216 The leaf node in each tree represents a pharmacological subtype of cancers, which is composed 217 of cells from the histological disparate tumour subtypes. We further employed the mRNA 218 expression profile and mutation/fusion profile to connect the molecular alterations with

219 pharmacological subtypes, delineating a novel perspective to re-classify the tumour according to 220 therapeutic response dependent of the intrinsic concordant molecular mechanism, by regardless 221 of tissues of origin. The cell similarity and the drug similarity were also redefined based on the 222 pharmacological subtypes.

\section{Pharmacological subtypes of cancers}

225 We built a pharmacological tree for each drug based on the divisibility of the drug sensitivity of 226 cancer cells. Taking the tree of the MEK1/2 inhibitor PD0325901 as an example (Fig. 1A), all cells 227 in the root node were first divided into left and right child nodes with relatively high and low 
228 sensitivity, respectively, and further cells in these two nodes were capable of being divided into 229 six final subgroups, C1, C2, C3, C4, C5, and C6. Each subgroup had varied drug sensitivity and 230 reached maximum divisibility, thus representing distinct pharmacological subtypes. C1 denotes 231 the most sensitive and C6 the most resistant subtype to the drug PD0325901. Except for C4 232 where both sensitive and resistant cells were mixed, the other subgroups contained the 233 homologous sensitive or resistant cells (Fig. 1B). There were much more sensitive cells in C1, C2 234 and C3 than resistant cells in C5 and C6 (Fig. 1C). We then investigated how histological 235 subtypes were distributed in the pharmacological subtypes (Fig. 1D). C1 was composed of 22 236 histological subtypes, with top 2 cancers, SKCM (19.6\%) and COREAD (11.3\%). C6 was 237 composed of 15 histological subtypes, with leading cancers including SCLC (36.6\%) and BRCA 238 (17.1\%).

240 Our analysis established 367 pharmacological trees. The number of pharmacological subtypes 241 with distinct drug sensitivities varied from 2 to 9 across 367 trees (Fig. 1E). This suggested that 242 heterogeneity of treatment effects across histological subtypes existed widely, and one 243 pharmacological subtype presenting concordant drug response indeed comprised histological 244 disparate cells to some extent. A high degree of treatment heterogeneity, meaning more than 245 seven pharmacological subtypes, was observed for $54.0 \%$ (16.9\% of the drugs had $7 ; 15.8 \%$ of 246 the drugs had 8 ; and $21.3 \%$ of the drugs had 9 pharmacological subtypes) of the drugs. These 247 drugs included Dabrafenib (BRAF inhibitor), Selumetinib (MEK1/2 inhibitor), Erlotinib (EGFR 248 inhibitor), and Alectinib (ALK inhibitor). 44.1\% of the drugs had 5 or 6 subtypes, showing a 249 moderate degree of treatment heterogeneity, while $1.9 \%$ of the drugs had subtypes lower than 4 , 250 showing a low degree of heterogeneity related to drug therapy (Fig. 1E).

252 We also particularly examined how pharmacological subtypes were constituted within the same 253 histological tumour subtypes, including LUAD, BRCA, SKCM, SCLC, COREAD and HNSC, in 254 which the average number of cell lines per drug was more than 35 (Fig. $1 \mathrm{~F}-\mathrm{H})$. We built 
255 approximately 310 pharmacological trees for each type of the above cancers. The density 256 distribution of the number of pharmacological subtypes indicated the varied treatment 257 heterogeneity across 6 histological subtypes (Fig. 1F). The shape of the distribution curve in 258 LUAD was characterized by double kurtosis and significantly positively biased, and oppositely the 259 curve in HNSC was negatively biased, suggesting the highest treatment heterogeneity in LUAD 260 and lowest treatment heterogeneity in HNSC. Furthermore, K-S test was applied to evaluate if 261 two different histological subtypes had the same level of treatment heterogeneity by comparing 262 their cumulative distributions. The results showed the cumulative distribution curve of LUAD is 263 significantly different from that of $\operatorname{HNSC}(p=1.11 \mathrm{e}-16$, K-S test) (Fig. 1G). The pairwise 264 comparisons between the curves of any two histological subtypes showed BRCA, SCLC, and 265 SKCM had the statistically similar distributions ( $p>0.05$, K-S test), and other cancers had not $266(p<0.05, \mathrm{~K}-\mathrm{S}$ test) (Supple. Fig. 1).

268 To observe the varied treatment heterogeneity of the different histological subtypes in detail, the 269 number of pharmacological subtypes of drugs across 6 histological cancers were shown in the 270 heatmap (Fig. 1H). Six histological tumors were arranged in columns according to their treatment 271 heterogeneity, from high to low. Interestingly we observed that EGFR inhibitors (Afatinib, 272 Cetuximab and Gefitinib) targeting in EGFR signalling pathway (green drugs in Fig. $1 \mathrm{H}$ ) were 273 clustered together $(p<0.001$, Fisher's exact test). And in LUAD and HNSC there were more 274 pharmacological subtypes of EGFR inhibitors than other drugs, suggesting high treatment 275 heterogeneity for EGFR inhibitors in LUAD and HNSC. In addition, drugs targeting ERK/MAPK 276 signalling (brown drugs in Fig. $1 \mathrm{H})$ were clustered together $(p<0.001$, Fisher's exact test). There 277 was high treatment heterogeneity for these drugs in SKCM.

\section{Genomic alterations associated with pharmacological subtypes}

280 To identify the molecular alterations associated with varied drug sensitivity, we examined whether 
281 the genomic alterations, including mutations and translocations, significantly changed across the 282 pharmacological subtypes. The statistical significance was determined by $p$ values calculated 283 with the Chi-squared test and corrected with the Benjamini-Hochberg method for controlling the 284 false discovery rate. If the pharmacological subtypes with higher drug sensitivity had more 285 frequent mutation or translocation events, we defined their association as a positive connection. If 286 the opposite was true, we defined their association as negative. Consequently, genes with 287 specific genomic alterations were associated with the respective drugs. Since one gene could 288 have multiple connected drugs and one drug could have multiple connected genes, these gene289 drug connections ultimately composed a network that allowed us to investigate the contribution of 290 either a drug or gene to the holistic understanding of how altered genes connect to drug 291 responses (Fig. 2A).

293 We first investigated the distribution of genes on their connected drugs (Fig. 2B). Our analysis 294 identified 462 genes whose genomic alterations were connected with at least two drugs' 295 pharmacological subtypes. Most of the genes (67.97\%) had fewer than 3 connections with drugs, 296 but $43(9.31 \%)$ genes whose genomic alterations were associated with more than 5 drugs. By 297 ranking genes by their number of connections to drugs, we found that the top ranked genes were 298 enriched in cancer genes (Fig. 2C; Supple. Fig. 2), a catalogue of genes with mutations that are 299 causally implicated in cancer provided by the COSMIC database [18]. The top 10 genes 300 connected to more than 9 drugs, $20 \%$ of which were remarkably annotated as cancer genes (Fig. $3012 \mathrm{C})$. These cancerous percentages for the top 10 ( $p=4.10 \mathrm{e}-2$, Fisher's exact test), top 20 $302(p=3.76 \mathrm{e}-3$, Fisher's exact test), and top 30 genes ( $p=1.64 \mathrm{e}-2$, Fisher's exact test) were 303 significantly higher than the background when using genome-wide 21,972 genes as a reference 304 (Fig. 2C). Among the top ranked oncogenes, BRAF ranked first with connections to 13 drugs, 305 followed by KRAS and TP53 with 11 and 9 drugs (Fig. 2D), implying that the highly drug-

306 connected genes that are not currently identified as cancer genes could actually be causally 307 implicated in cancer therapy, such as ADK-VCL fusion, TNFRSF9 and LTB, which showed 
308 connections to over 20 drugs and ranked as the top five genes (Supple. Fig. 2).

310 We then investigated the distribution of drugs on their connected genes. For the majority of drugs

311 (61.64\%), the number of connected genes ranged from 2 to 10 (Fig. 2E). There was the same

312 number of genes that showed a positive connection as that showed a negative connection (Fig.

313 2F). We ranked the drugs with more than 50 genes' connection with their pharmacological

314 subtypes (Fig. 2G). Drugs with gene connections greater than 50 included PHA-665752,

315 affecting the RTK signaling pathway, Veliparib, affecting the genome integrity pathway, Obatoclax

316 Mesylate, affecting the apoptosis regulation pathway, and AZD2014, affecting the PI3K/MTOR

317 signaling pathway.

319 Next, we observed how the mutation rate of genes associated with the variability of drug 320 sensitivity changed among drug-sensitive and drug-resistant subtypes. Taking the MEK $1 / 2$ 321 inhibitor PD0325901 as an example, five genomic alterations were found to connect to the drug 322 and be associated with pharmacological subtypes. As shown in Fig. 2H, BRAF mutations and 323 KRAS mutations occurred significantly more frequently (over 20\%) in the sensitive subtype C1 324 and then decreased gradually in the subtypes C2, C3, C5 and C6. Here one subtype C4 with 325 sensitive and resistant cells mixed was excluded in the analysis. Conversely, RB1 mutation, 326 FRG1BP mutation and RAB10 mutation occurred more frequently in the resistant subtypes. 327 Therefore, C1 group, consisting mainly of SKCM (19.6\%) and COREAD (Fig. 1D), was 328 significantly sensitive to PD0325901 due to its high occurrence of BRAF and KRAS mutations 329 and low occurrence of RB1, FRG1BP and RAB10 mutations (Fig. 2H). Other types of MEK 330 inhibitors, including Refametinib, Trametinib, Selumetinib, and $\mathrm{Cl}-1040$, further confirmed that 331 BRAF, KRAS, and RB1 mutations were robustly connected to pharmacological subtypes, 332 contributing to the drug sensitivity of MEK inhibitors (Supple. Fig. 3). Our analysis revealed that 333 the varied distribution of genomic alterations across pharmacological subtypes could lead to their 334 differences in response to anticancer therapies. 


\section{Expression alterations associated with pharmacological subtypes}

337 We also associated mRNA expression with the pharmacological subtypes. The connection

338 between gene expression and pharmacological subtypes was determined by $p$ values calculated

339 with the Kruskal-Wallis $\mathrm{H}$ test and corrected with the Benjamini-Hochberg method for controlling

340 the false discovery rate. If the subtypes with high drug sensitivity had higher gene expression, we

341 defined this connection as positive. If the opposite was true, we defined the connection as

342 negative. The connections between genes and drugs constituted a network (Fig. 3A). Since some

343 genes whose expression was connected to pharmacological subtypes were indirectly associated

344 with drug sensitivity, we then removed genes that had functions outside of the core cancer

345 pathways (Supple. Tab. 1).

347 We first investigated the distribution of genes on their connected drugs (Fig. 3B). Our analysis

348 identified 1,357 genes whose expression was connected to at least two drugs' pharmacological 349 subtype. Among them, $16.14 \%, 37.07 \%$ and $21.30 \%$ of genes respectively had connections with

350 greater than 50, 20 and 10 drugs. The top 10 genes connected to more than 143 drugs, $30 \%$ of 351 which were annotated as cancer genes (Fig. 3C; Supple. Fig. 4). These cancerous percentages 352 for the top 10 ( $p=3.62 \mathrm{e}-2$, Fisher's exact test), top 20 ( $p=3.67 \mathrm{e}-3$, Fisher's exact test), and top 30

353 genes ( $p=3.90 \mathrm{e}-4$, Fisher's exact test) were significantly higher than the background. Comparing 354 with genomic alterations, each of genes connected to more drugs and the top genes had higher 355 cancerous percentages. The top genes with their expression alterations associated with subtypes 356 were different from those with genomic alterations associated with subtypes (Fig. 3D, Fig. 2D).

357 Among them, IKZF1 and IL21R ranked the top two with connections to 164 drugs and 155 drugs

358 (Fig. 3D).

360 We then investigated the distribution of drugs on their connected genes. The number of genes 
361 whose expression was associated with the subtypes varied from 0 to 700 across the drugs, with $36214.53 \%$ drugs had more than 200 gene connections (Fig. 3E). Moreover, genes whose

363 expression was positively connected with drug resistance across pharmacological subtypes were

364 significantly more abundant than genes that were negatively connected (Fig. 3F). The top 20

365 drugs with the most connections to genes included UNC1215, affecting the chromatin, NPK76,

366 affecting cell cycle, Methotrexate, affecting DNA replication, AKT inhibitor VIII, affecting

367 PI3K/MTOR signaling pathway, YM201636, affecting PI3K/MTOR signaling pathway,

368 NPK76-II-72-1, affecting the cell cycle pathway, and some compounds such as Zibotentan, A-

369484954 et al, affecting multiple or unknown pathways (Fig. 3G).

370

371 Methotrexate, as an example, is a chemotherapy that specifically acts during DNA and RNA 372 synthesis, and cancer cells were classified into seven subtypes (Fig. $3 \mathrm{H}$ ) in terms of drug 373 sensitivity to methotrexate. The first subtype, $\mathrm{C} 1$, was most sensitive to the drug, and the seventh 374 subtype, C7, was the most resistant to the drug (Fig. 3H). C1 included 12 histological subtypes, 375 with the top 2 which shown in the bottom of Fig. 3H, ALL and DLBC. C2 and C3 were composed 376 of 18 and 19 histological subtypes, respectively, with SCLC both included in the top 2 (Fig. 3H). A 377 total of 297 cancer genes with high expression in sensitive group (C1, C2 and C3) and low 378 expression in resistant group ( $\mathrm{C} 4, \mathrm{C} 5, \mathrm{C} 6$ and $\mathrm{C} 7)$, which had positive connections to 379 pharmacological subtypes of methotrexate, were selected (Fig. 3I). Pathway enrichment analysis 380 showed that these genes were enriched in pathways including cell cycle and DNA replication et al. 381 (Fig. 3J), suggesting that the ectopic activation of cell cycle lead to the sensitive response of ALL, 382 DLBC, LAML and SLCL to Methotrexate.

\section{Redefining the similarity of cells based on pharmacological subtypes}

385 Pharmacological subtypes provide us a new point of view to redefine the similarity of cells purely 386 from therapeutic concordance. Thus we calculated the similarity of pairwise cells based on 
387 pharmacological subtypes of drugs. If there are quite a few cases that two cells are in the same 388 sensitive or resistant pharmacological subtypes of drugs, these cells gain a high similarity.

389 Otherwise, they gain a low similarity. The similarity of cells defined a hierarchical cluster as 390 shown in Fig. 4A. We further applied the Fisher's exact test to observe if the cells in the same 391 clusters were from the same histological subtypes (Fig. 4B-D). The points with black border in the 392 figure represent statistical significance $(p<0.05)$, with the smaller $p$ values shown in red.

394 The results showed that the cancer cell lines were respectively divided into 2, 4 and 15 clusters at 395 the different hierarchical levels (Fig. 4A). Two clusters at the high level were reflected by two 396 distinct patterns of similarity (Fig. 4A). One cluster (Cluster H1) was significantly correlated with 397 solid tumors including BRCA, COREAD, LUAD, MESO, OV, and PAAD, and the other (Cluster H2) 398 hematological tumors including ALL, DLBC, LAML, LCML and MM (Fig. 4B). So in a holistic view, 399 the different types of hematological tumors shared a similar drug sensitivity profile, which was 400 quite different from the profile of solid tumors. However, for a given cancer type (the right panel of 401 Fig. 4B), such as ACC, BLCA, CESC, CLL, or ESCA et al., its cells were unbiasedly distributed in 402 two clusters, showing the treatment heterogeneity for the cells from the same tissue origin. At the 403 median hierarchical levels, 4 clusters were identified (Fig. 4C). Among them, Cluster M3 was 404 specifically associated with haematological tumors ( $p<0.001$, Fisher's exact test) including ALL, 405 DLBC, LAML, LCML and MM. The treatment heterogeneity was still observed in cancers.

407 When cell lines were divided into 15 clusters at the lower hierarchical level (Fig. 4D), we observed 408 that the cells from ACC, CLL, STAD, or UCEC were unbiasedly distributed in the different clusters, 409 still showing their treatment heterogeneity. Interestingly, the cells from some cancers such as 410 HNSC, KIRC, and SCLC were enriched in more than 2 clusters with distinctly different drug 411 response. For instance, HNSC cells were enriched in Clusters L12, L4 and L5; KIRC cells were 412 enriched in Clusters L15 and L4; SCLC cells were enriched in Clusters L13, L3 and L7. 413 Interestingly, the SCLC cells in Cluster L13 shared a more similar profile with hematological 
414 tumors including DLBC, LAML and LCML. To investigate the underlying mechanism of such

415 treatment heterogeneity in SCLC, we applied DESeq2 analysis to call the differentially expressed

416 genes between Cluster L13 and Cluster L3, and further identified the differential functions via

417 gene set enrichment analysis (GSEA) [16]. The results showed that the functions like DNA 418 replication, RNA transport et al. were significantly upregulated in Cluster L13 cells, while in

419 Cluster L3 cells the functions like immune response, antigen processing and presentation et al.

420 were mainly upregulated (Supple. Fig. 5A). Moreover, MYC, as an oncogenic transcription factor

421 of cell growth and proliferation via enhancing the cell cycle regulated genes [19], was significantly

422 upregulated in Cluster L13 than in L3, with a log2-fold change of 4.28 (Supple. Fig. 5B). These

423 suggested that SCLC cell lines in Cluster L13 were more malignant than those in Cluster L3.

\section{Redefining the similarity of drugs based on pharmacological subtypes}

426 The similarity of pairwise drugs was also defined as the summarized similarity of drug response 427 across the same cells which were tested for both drugs. For two drugs, if there are quite a few of 428 cell pairs which are in the sensitive (or resistant) pharmacological subtypes of one drug are also 429 in the sensitive (or resistant) subtypes of the other drug, they gain a high similarity. Otherwise, 430 they gain a low similarity. We then generated a drug similarity profile across 367 drugs, and 431 similarly, a hierarchical clustering algorithm was used to group drugs based on drug similarity (Fig. 432 4E). The results showed that at the high level, drugs were divided into two clusters, 433 demonstrating two different similarity patterns. The first cluster (Cluster H1) mostly included 434 chemotherapy drugs such as those effecting in DNA replication and mitosis, and the second 435 cluster was mixed with target and chemotherapy drugs, specifically enriched by the drugs on RTK 436 signaling, other kinases, and cytoskeleton (Fig. 4F). When the drugs were divided into 4 clusters

437 (Fig. 4E, G), we observed that the drugs enriched in L2 included chemotherapy drugs effecting in 438 cell cycle, chromatin histone accelylation, and DNA replication, the drugs enriched in L3 were 439 functioning in PI3K/mTOR signaling pathway, and the drugs enriched in L4 were hormone related 440 ones. Moreover, L4, accounting for a massive part of drugs, also included the drugs targeting the 
441 different types of signaling pathways such as ABL, EGFR, Jak and P38, RTK and WNT signalling.

442 These drugs did not reach the significant level but provided the obvious association.

443

444 We further observed the number of pharmacological subtypes of these drugs that were arranged

445 by the clustering structure above (Fig. $4 \mathrm{H}$ ). Remarkably, there was the varied treatment

446 heterogeneity for the drugs in the different clusters. The drugs in Cluster L4 had more subtypes

447 but those in Cluster L1 and L3 had less subtypes, suggesting higher treatment heterogeneity for

$448 \quad \mathrm{~L} 4$ and lower treatment heterogeneity for L1 and L3.

\section{An access to pharmacological subtypes of drugs}

451 We developed a website to store and visualize the pharmacological subtypes of drugs, as well as

452 the distributions of histological subtypes across them, and connected genomic and expression 453 alterations. All data and results used for the study are easily accessible via 454 http://www.hywanglab.cn/dtdb/ (Supple. Fig. 6).

\section{Discussion}

457 In this study, we integrated drug response information to pharmacologically reclassify tumour 458 subtypes, as well as identify intrinsic concordant molecular mechanisms. Unlike the many studies 459 that have aimed to unravel cancer heterogeneity by defining subtypes within the same tissues, 460 our study aimed to systematically uncover that cancers across disparate tissues of origin can 461 belong to one pharmacological subtype, benefiting from similar anticancer therapies, because 462 they share a common molecular mechanism of oncogenesis. Besides, we also developed the 463 new measures to redefine cell similarity and drug similarity from the therapeutic concordance, 464 which provided a new point of view to study cancer heterogeneity. The similarity of cells further 465 depicted that cells from the different origin of tissue could share the similar responses of drugs; 
466 likewise, that cells from the same origin of tissue could have distinct drug responses, thus 467 indicating the new subtypes.

469 Our analysis identified, for instance, that although SKCM and COREAD are histologically different, 470 both belonged to one pharmacological subtype, the MEK1/2 inhibitor C1 subtype, and were 471 similarly sensitive to MEK1/2 inhibitors (Fig. 1A-D). By connecting genomic alterations with 472 pharmacological subtypes, mutations in the oncogene BRAF or KRAS were found to be 473 overwhelmingly more frequent in the $\mathrm{C} 1$ group (Fig. $2 \mathrm{H}$ ). BRAF and KRAS are the kinases 474 upstream of MEK1/2 that transmits the signals down through MEK1/2 without other major 475 signalling branches [20,21]. Our results illustrated that MEK inhibitors were able to effectively 476 block aberrantly activated signals from BRAF or KRAS mutations. Further investigation by 477 cBioportal (https://www.cbioportal.org/) of TCGA patients also revealed that approximately $60 \%$ of 478 THCA (thyroid carcinomas) and 50\% of SKCM (melanomas) harboured BRAF mutations, and $65 \%$ 479 of PAAD (pancreas) and 40\% COREAD (colorectal carcinomas) possessed KRAS mutations, a 480 rate that was strikingly higher than that of other cancers (Supple. Fig. 7A, B). Given the high 481 mutant frequency of BRAF in THCA and SKCM, and KRAS in PAAD and COREAD, such 4 482 cancer types were significantly enriched in C1 pharmacological subtype of MEK1/2 inhibitor 483 PD0325901 (THCA, $p=8.79 \mathrm{e}-4$; SKCM, $p=1.30 \mathrm{e}-14$; PAAD, $p=4.17 \mathrm{e}-3$; COREAD, $p=4.58 \mathrm{e}-4$; 484 Hypergeometric Distribution Test) (Supple. Tab. 2). In contrast, mutations in the oncogenes RB1 485 was found to be less frequent in the $\mathrm{C} 1$ group than in other groups. RB1 is a negative regulator of 486 the cell cycle, with its active hypophosphorylated form binding the transcription factor E2F1 [22].

487 RB1 mutations lead to the ectopic activation of the cell cycle, which cannot be controlled using 488 MEK inhibitors. This may be because RB1, located downstream of MEK1/2, gain ectopic 489 activation independent of upstream stimulation. In another instance, therapeutic concordance 490 was identified across histologically disparate blood cancers in regard to methotrexate, a 491 chemotherapy drug specially acting during DNA and RNA synthesis (Fig. 3H). This is well known 492 that Methotrexate is used in haematological malignancies but less so in solid cancers [23]. 
493 Functional analysis of the positively correlated genes showed that haematological tumors had 494 more active gene expression related to the cell cycle than solid tumours (Fig. 3I, J). The 495 molecular basis of such extraordinary activation of the cell cycle in haematological tumors could 496 explain their good response to chemotherapy drugs, which work mainly by inhibiting mitosis and 497 cell division. This demonstrates pharmacological subtypes benefiting from similar anticancer 498 therapies can be due to common molecular mechanisms related to tissue-specific gene 499 expression and pathways. Our website provided the pharmacological subtypes of 367 drugs as 500 well as their connections with the molecular alterations for the users to retrieve and download.

502 We also investigated the treatment heterogeneity for six types of histological cancers, including 503 LUAD, BRCA, SKCM, SCLC, COREAD and HNSC. High treatment heterogeneity in LUAD was 504 observed compared with other types of cancers, suggesting that anticancer therapies for LUAD 505 should be more complicated than those for other types of cancers (Fig. 1F-H). Moreover, the 506 increased number of subtypes of EGFR inhibitors in LUAD indicated there was high treatment 507 heterogeneity when EGFR inhibitors were applied into the therapies of LUAD patients (Fig. 1H).

509 Our analysis showed that genes whose genomic/expression alterations frequently connect to 510 anticancer drugs had a higher likelihood of being cancer genes. At genomic level, the top 10 511 genes were connected to more than 9 drugs, $20 \%$ of which (including BRAF and KRAS) were 512 annotated as cancer censor genes. At expression level, each of genes connected to more drugs 513 and the top genes had higher cancerous percentages. This indicated that some genes currently 514 not identified as cancer genes could actually be causally implicated in cancer. In our analysis, 515 ADK-VCL, TNFRSF9 and LTB, as the top three most connected genes, were identified as novel 516 cancer genes for further investigation. Adenosine kinase (ADK) has an important role in mitosis, 517 tumorigenesis, tumor-associated tissue remodeling, and invasion, and manipulation of ADK has 518 the potential to be a therapeutic strategy for invasive breast cancer [24]. Vinculin (VCL) is a 519 cytoskeletal protein associated with cell-cell and cell-matrix junctions, which interacts with many 
520 focal adhesion proteins and has been found to be closely linked to tumor migration [25]. ADK-

521 VCL had 45 drug connections suggesting its therapeutic potential, although ADK-VCL fusion

522 events were rarely reported. We speculated that its fusion was beneficial for tumor metastasis,

523 but evidence was lacking. The tumor necrosis factor receptor superfamily member 9 (TNFRSF9)

524 with connections to 25 drugs, also known as 4-1BB and CD137, is an immune co-stimulatory

525 receptor. TNFRSF9 is expressed on activated immune cells including natural killer (NK) cells,

526 effector $\mathrm{T}$ cells and antigen presenting cells, among them dendritic cells, macrophages, and B

527 cells [26]. LTB (Lymphotoxin Beta), with connections to 20 drugs, is a type II membrane protein of

528 the TNF family. Giuseppina et al. found that a decrease in lymphotoxin- $\beta$ production by tumor

529 cells was associated with a loss of follicular dendritic cell phenotype and diffuse growth of

530 follicular lymphomas [27]. Recently, the gene was found to be associated with immune infiltration

531 of breast and endometrial cancer tumors [28, 29]. The analysis using the TCGA dataset showed

532 that TNFRSF9 was altered in approximately $5 \%$ of cases of Cholangiocarcinoma, adrenocortical

533 carcinoma (ACC) and lymphoid neoplasm diffuse large B-cell lymphoma (DLBC), with deep

534 deletion being the dominant alteration (Supple. Fig. 8A). In addition, more than $14 \%$ of DLBC

535 patients had altered LTB genes, with mutations and deletion being the major factors (Supple. Fig.

536 8B). Therefore, the importance of the roles of these genes in cancers could be underappreciated.

538 Genes whose alterations were positively connected with drug resistance were significantly more

539 frequent than those negatively connected at the mRNA expression level but not at the genomic

540 level (Fig. 3F). A positive connection meant that genomic alterations or high expression indicated

541 increased drug sensitivity. In this way, genes with a positive connection could be indicators of

542 therapeutic efficiency for connected drugs. Genes with negative connections could be potential

543 biomarkers for developing new therapies to overcome the resistance of connected drugs. Our

544 analysis suggests that most of the genes whose expressions are altered during cancer initiation

545 and development (driver genes or not) are located in ectopically activated pathways that could be

546 controlled by anticancer drugs. Only a proportion of altered genes are outside of the cellular 
547 pathways targeted by anticancer drugs, and their alterations may maintain the growth signals of 548 cancer cells when anticancer drugs are used. Therefore, these genes may be potential 549 therapeutic biomarkers for overcoming drug resistance to the connected drugs.

551 Based on pharmacological subtypes, the cell similarity and the drug similarity could be re-defined

552 (Fig. 4). The cells from the same origin of tissue can be dispersed in the different pharmacological 553 subtypes, showing the completely different response to the same drug, because of their 554 intrinsically molecular heterogeneity. For instance, HNSC, KIRC and SLCL cells were separated 555 into more than two clusters with distinct drug response (Fig. 4D). Specifically, the SCLC cells with 556 more malignant signatures, in particular, higher expression levels of MYC and upregulated 557 pathways involving DNA replication and cell cycle, were far away from the other SCLC cells and 558 close to hematological tumors, instead. Interestingly, by connecting to expression alterations, our 559 analysis further confirmed that SCLC cells more like hematological tumors had the characteristic 560 of the ectopic activation of cell cycle, which led to their sensitive to a chemotherapy Methotrexate 561 (Fig. 3H-J). Our pharmacological analysis has recognized a new subtype of SCLC cells, with the 562 high expression of an oncogenic transcription factor MYC as a marker, can benefit from 563 chemotherapy than other SCLC subtypes. This finding was consistent with the latest studies [30, 564 31]. Therefore, the subtypes identified by re-classifying the same origin of tissue using 565 pharmacological data are worth to be further investigated. In addition, our analysis of the drug 566 similarity unraveled that the drugs were categorized into two major groups, wherein the 567 chemotherapeutic drugs tended to share the similar pattern (Fig. 4E, F). Moreover, Cluster L4 568 including many drugs targeting the signalling pathways had more pharmacological subtypes, 569 suggesting a high treatment heterogeneity for some target drugs (Fig. 4H).

\section{Conclusions}


572 In summary, our study was a systematic analysis which aimed to reveal intrinsic therapeutic

573 concordance across histologically disparate tumour subtypes. The findings provide us with a

574 purely therapy-oriented perspective to re-examine tumour classifications independent of histology

575 subtypes.

576 


\section{Conflict of interest}

578 The authors declare no competing financial interests.

579

580 Availability of data and materials

581 The data and materials that supporting the conclusion of this paper have been included within the

582 article and the website (www.hywanglab.cn/dtdb/).

583

\section{Acknowledgements}

585 This work was supported by grants from the National Key Research and Development Program

586 (2017YFC0908500 to HW) and the National Natural Science Foundation of China (31771469 and 58731571363 to HW; 81772465 to $\mathrm{FZ})$.

588

589 Authors' contributions

590 HW conceived the hypothesis. YX, JZ and HW designed and performed the data analysis. ZC,

591 WL, YD, XC and TW collected and preprocessed the data. HW, YX, JZ, JK and FZ wrote and 592 revised the manuscript. 


\section{References}

594 1. Swanton, C., Intratumor heterogeneity: evolution through space and time. Cancer Res, 595 2012. 72(19): p. 4875-82.

5962 2. Bedard, P.L., et al., Tumour heterogeneity in the clinic. Nature, 2013. 501(7467): p. 355-

597

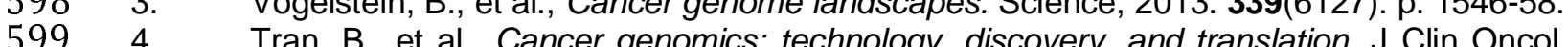

$600 \quad$ 2012. 30(6): p. 647-60.

601 5. Chapman, P.B., et al., Improved survival with vemurafenib in melanoma with BRAF

602

603

604

605

606

607

608

609

610

611

612

613

614

615

616

617

618

619

620

621

622

623

624

625

626

627

628

629

630

631

632

633

634

635

636

637

638

639

640

641

642

643

644

645

V600E mutation. N Engl J Med, 2011. 364(26): p. 2507-16.

6. Mok, T.S., et al., Gefitinib or carboplatin-paclitaxel in pulmonary adenocarcinoma. N Engl J Med, 2009. 361(10): p. 947-57.

7. Perou, C.M., et al., Molecular portraits of human breast tumours. Nature, 2000. 406(6797): p. 747-52.

8. Cancer Genome Atlas, N., Comprehensive molecular portraits of human breast tumours. Nature, 2012. 490(7418): p. 61-70.

9. Prat, A., et al., PAM50 assay and the three-gene model for identifying the major and clinically relevant molecular subtypes of breast cancer. Breast Cancer Res Treat, 2012. 135(1): p. 301-6.

10. Schmitz, R., et al., Genetics and Pathogenesis of Diffuse Large B-Cell Lymphoma. N Engl J Med, 2018. 378(15): p. 1396-1407.

11. Cancer Genome Atlas Research, N., et al., The Cancer Genome Atlas Pan-Cancer analysis project. Nat Genet, 2013. 45(10): p. 1113-20.

12. Hoadley, K.A., et al., Multiplatform analysis of 12 cancer types reveals molecular classification within and across tissues of origin. Cell, 2014. 158(4): p. 929-944.

13. Drilon, A., et al., Efficacy of Larotrectinib in TRK Fusion-Positive Cancers in Adults and Children. N Engl J Med, 2018. 378(8): p. 731-739.

14. Garnett, M.J., et al., Systematic identification of genomic markers of drug sensitivity in cancer cells. Nature, 2012. 483(7391): p. 570-5.

15. Benjamini, Y. and Y. Hochberg, Controlling the False Discovery Rate: A Practical and Powerful Approach to Multiple Testing. Journal of the Royal Statistical Society: Series B (Methodological), 1995. 57(1): p. 289-300.

16. Subramanian, A., et al., Gene set enrichment analysis: a knowledge-based approach for interpreting genome-wide expression profiles. Proc Natl Acad Sci U S A, 2005. 102(43): p. $15545-50$.

17. Love, M.I., W. Huber, and S. Anders, Moderated estimation of fold change and dispersion for RNA-seq data with DESeq2. Genome Biol, 2014. 15(12): p. 550.

18. Tate, J.G., et al., COSMIC: the Catalogue Of Somatic Mutations In Cancer. Nucleic Acids Res, 2019. 47(D1): p. D941-d947.

19. Oster, S.K., et al., The myc oncogene: Marvelous/Y Complex. Adv Cancer Res, 2002. 84: p. 81-154.

20. McCubrey, J.A., et al., Roles of the Raf/MEK/ERK pathway in cell growth, malignant transformation and drug resistance. Biochim Biophys Acta, 2007. 1773(8): p. 1263-84.

21. Simanshu, D.K., D.V. Nissley, and F. McCormick, RAS Proteins and Their Regulators in Human Disease. Cell, 2017. 170(1): p. 17-33.

22. Chen, H.Z., S.Y. Tsai, and G. Leone, Emerging roles of E2Fs in cancer: an exit from cell cycle control. Nat Rev Cancer, 2009. 9(11): p. 785-97.

23. Koźmiński, P., et al., Overview of Dual-Acting Drug Methotrexate in Different Neurological Diseases, Autoimmune Pathologies and Cancers. Int J Mol Sci, 2020. 21(10).

24. Shamloo, B., et al., Dysregulation of adenosine kinase isoforms in breast cancer. Oncotarget, 2019. 10(68): p. 7238-7250.

25. de Semir, D., et al., PHIP drives glioblastoma motility and invasion by regulating the focal adhesion complex. Proc Natl Acad Sci U S A, 2020. 117(16): p. 9064-9073. 
646 26. Fröhlich, A., et al., Comprehensive analysis of tumor necrosis factor receptor TNFRSF9 (4-1BB) DNA methylation with regard to molecular and clinicopathological features, immune infiltrates, and response prediction to immunotherapy in melanoma. EBioMedicine, 2020. 52: p. 102647.

27. Pepe, G., et al., Reduced lymphotoxin-beta production by tumour cells is associated with loss of follicular dendritic cell phenotype and diffuse growth in follicular lymphoma. $\mathrm{J}$ Pathol Clin Res, 2018. 4(2): p. 124-134.

28. Terkelsen, T., et al., Secreted breast tumor interstitial fluid microRNAs and their target genes are associated with triple-negative breast cancer, tumor grade, and immune infiltration. Breast Cancer Res Treat, 2020. 22(1): p. 73.

29. Ding, H., et al., Prognostic Implications of Immune-Related Genes' (IRGs) Signature Models in Cervical Cancer and Endometrial Cancer. Front Genet, 2020. 11: p. 725.

30. Bian, X., et al., The MYC Paralog-PARP1 Axis as a Potential Therapeutic Target in MYC Paralog-Activated Small Cell Lung Cancer. Front Oncol, 2020. 10: p. 565820.

31. Ireland, A.S., et al., MYC Drives Temporal Evolution of Small Cell Lung Cancer Subtypes by Reprogramming Neuroendocrine Fate. Cancer Cell, 2020. 38(1): p. 60-78.e12.

662

\section{Figures and Tables Legends}

665 Figure 1. Re-classifying cancers to generate pharmacological subtypes and drug 666 sensitivity profiles. (A) Pharmacological subtype tree of the drug PD0325901. Cancer cells

667 were classified into six pharmacological subtypes based on their drug sensitivity to MEK inhibitor

668 PD0325901. (B) The boxplot shows the drug sensitivities of six subtypes (leaf nodes): C1, C2, C3,

669 C4, C5, and C6, indicating a gradually increasing degree of resistance to the drug PD0325901.

670 Blue points refer to cell lines sensitive to the drug, while red points resistant. (C) The barplot

671 shows the number of cell lines in each subtype. (D) The pie chart shows the distribution of

672 histological subtypes across pharmacological subtypes. (E) The pie chart illustrates the numerical

673 proportion of different degrees of the furthest divisible pharmacological subtypes. Cancer cells

674 could be maximally classified into up to 9 subtypes for different drugs. Among them, 7-9 subtypes

675 accounted for more than half of drugs. (F) The density of the number of pharmacological

676 subtypes indicates the varied treatment heterogeneity across six histological cancers, in which

677 the average number of cell lines among all drugs are over 35. (G) The empirical cumulative

678 distribution function visualizes the density of drug subtypes in LUAD vs. HNSC. P value was

679 calculated by using the Kolmogorov-Smirnov test (K-S test). $(\mathrm{H})$ Hierarchical clustering of the

680 number of pharmacological subtypes across six cancers. Each row in the heatmap represents a 
681 drug, and each cell in the heatmap represents the number of subtypes of the cancer in the drug.

682 The red colour indicates more subtypes, and the blue colour less subtypes.

684 Figure 2. Genomic alterations determine the various drug sensitivities across

685 pharmacological subtypes. (A) A gene with its genomic alteration significantly changed across

686 the pharmacological subtypes of a drug is linked with the drug, generating a gene-drug network. A 687 positive connection meant that subtypes with high drug sensitivity had a more frequent 688 occurrence of genomic alterations. Conversely, those that were less frequent were defined as 689 negative connections. (B) $9.31 \%$ genes had more than 5 connected drugs. In addition, $67.97 \%$ of 690 genes had fewer than 3 connected drugs. (C) The percentage of cancer genes in the top 10, 20, 691 and 30 genes, and the genome-wide 21,972 genes were referred to as background. (D) The top 692 cancer censor genes defined by COSMIC and the corresponding number of drug connections. (E) 693 When considering the genes connected to the drugs, $5.17 \%$ of drugs have more than 50 694 connected cancer genes. In addition, $61.64 \%$ of drugs had fewer than 10 connected cancer 695 genes. (F) Comparison of the number of genes with a positive connection with the number of 696 genes with a negative connection. (G) Drugs with cancer gene connections greater than 50 were 697 ranked. (H) BRAF mutation, RB1 mutation, KRAS mutation, FRG1BP mutation and RAB10 698 mutation were associated with the five pharmacological subtypes of PD0325901. Histograms 699 show the mutation/translocation ratio of the five genes in the five pharmacological subtypes of 700 PD0325901.

702 Figure 3. Tissue-specific gene expression determines the various drug sensitivities across 703 pharmacological subtypes. (A) A gene with its expression significantly changed across the 704 pharmacological subtypes of a drug is linked with this drug, generating a gene-drug network. A 705 positive connection meant that subtypes with high drug sensitivity had higher gene expression. 706 Conversely, those with low expression were defined as negative connections. (B) Pie chart 707 illustrating the numerical proportion of the number of connected drugs with genes whose 
708 expression was associated with the pharmacological subtypes. A total of $16.14 \%$ of genes had 709 more than 50 connected drugs. In addition, $25.50 \%$ of genes had less than 10 connected drugs.

710 (C) The percentage of cancer genes in the top 10, 20, and 30 genes, and the total cancer genes

711 were referred to as background. (D) The top cancer censor genes defined by COSMIC and the 712 corresponding number of drug connections. (E) Distribution of the genes functioning in the core 713 cancer pathways whose expression was connected with pharmacological subtypes. A total of $71414.53 \%$ of drugs had more than 200 connected genes. $(F)$ The number of genes with positive 715 connections was significantly greater than the number of genes with negative connections. $(G)$

716 Top 20 drugs with the most connections to genes. (H) Seven subtypes, C1, C2, C3, C4, C5, C6 717 and $\mathrm{C}$, had varied drug sensitivity to methotrexate, with $\mathrm{C} 1$ being the most sensitive to the drug 718 and $\mathrm{C} 7$ being the most resistant to the drug. The boxplot below shows the drug sensitivities of the 719 three subtypes (leaf nodes) C1, C2, C3, C4, C5, C6 and C7, indicating a gradually increasing 720 degree of resistance to the drug. (I) A total of 297 genes were highly expressed in sensitive group 721 (C1, C2 and C3) and lowly expressed in resistant group (C5, C6 and C7). (J) Pathways enriched 722 by the 297 highly expressed genes in sensitive group (C1, C2 and C3).

724 Figure 4. Cell similarity and drug similarity based on pharmacological subtypes. (A) 725 Hierarchical clustering of a cell similarity matrix which was generated based on drug response of 726 cells to drugs, with the red colour indicating high similarity, and the blue colour indicates low 727 similarity between two cell lines. Clusters were formed by partitioning of the dendrogram at a high, 728 median and low height respectively. (B) Two clusters $(\mathrm{H} 1, \mathrm{H} 2)$ were formed by cutting the 729 dendrogram at a height of 30. Points with black border represents statistical significance $(p<0.05)$ 730 determined by Fisher's exact test. Almost all histological subtypes of blood cancer were grouped 731 together. (C) Four clusters (M1, M2, M3 and M4) were formed by cutting the dendrogram at a 732 height of 15. (D) Fifteen clusters (L1-15) were formed by cutting the dendrogram at a height of 5. 733 Cancers with the same tissue of origin and organ did not cluster together. (E) Hierarchical 734 clustering of the drug similarity profiles with the red colour indicating a high similarity and the blue 
735 colour indicating a low level. Clusters were formed by partitioning of the dendrogram at a high 736 and low height respectively. $(\mathrm{F})$ Two clusters $(\mathrm{H} 1, \mathrm{H} 2)$ were formed by cutting the dendrogram at 737 a height of 30 . Points with black border represents statistical significance $(p<0.05)$ determined

738 by Fisher's exact test. Targeted therapy related drugs were grouped together. (G) Four clusters 739 (L1, L2, L3 and L4) were formed by cutting the dendrogram at a height of 5. Cytoskeleton, EGFR 740 signaling, Hormone-related and WNT signalling pathways cluster together. $(H)$ The line chart 741 illustrates the number of subtypes of each drug. 


\section{Supplementary Data}

743 Supplementary Figure 1. The comparisons between two types of cancers by the numbers

744 of pharmacological subtypes across drugs.

745

746 Supplementary Figure 2. The top $\mathbf{3 0}$ genes whose genomic alterations frequently connect

747 to pharmacological subtypes.

748

749 Supplementary Figure 3. Genomic alterations connect to pharmacological subtypes based

750 on MEK inhibitors, Refametinib, Trametinib, Selumetinib, PD-0325901, and CI-1040. BRAF

751 mutation and KRAS mutation were positively connected to 5 and 4 drugs, respectively. RB1

752 mutation had a negative connection to 5 drugs.

753

754 Supplementary Figure 4. The top 30 genes whose expressions frequently connect to 755 pharmacological subtypes.

756

757 Supplementary Figure 5. Pathways significantly enriched in the comparison of Cluster L13

758 vs. L3 and the different levels of MYC expression in such comparison.

760 Supplementary Figure 6. Introduction of the website.

762 Supplementary Figure 7. Mutation frequency of BRAF and KRAS in pan-cancer patients in

763 the TCGA dataset. (A) $60 \%$ of thyroid carcinomas and $50 \%$ of melanomas harboured BRAF

764 mutation. (B) $65 \%$ of pancreas and $40 \%$ colorectal carcinomas harboured KRAS mutation. 
766 Supplementary Figure 8. Mutation frequency of TNFRSF9 and LTB in pan-cancer patients

767 in the TCGA dataset. (A) TNFRSF9 was altered in cholangiocarcinoma, lymphoid neoplasm

768 diffuse large B-cell lymphoma, adrenocortical carcinoma, etc. in approximately $5 \%$ of cases, with

769 deep deletion being the dominant alteration. (B) More than 14\% of DLBC patients had altered

$770 \quad$ LTB genes.

771

772 Supplementary Table 1. Genes mapped to core cancer pathways.

773

774 Supplementary Table 2. The enrichment of histological cancers in the most sensitive 775 pharmacological subtype of PD0325901. 


\section{Abbreviations}

777 ACC Adrenocortical carcinoma

778 ALL Acute lymphoblastic leukemia

779 BLCA Bladder urothelial carcinoma

780 BRCA Breast invasive carcinoma

781 CESC Cervical squamous cell carcinoma and endocervical adenocarcinoma

782 CLL Chronic lymphocytic leukemia

783 COREAD Colon adenocarcinoma and rectum adenocarcinoma

784 DLBC Lymphoid neoplasm diffuse large B-cell Lymphoma

785 ESCA Esophageal carcinoma

786 GBM Glioblastoma multiforme

787 HNSC Head and neck squamous cell carcinoma

788 KIRC Kidney renal clear cell carcinoma

789 LAML Acute myeloid leukemia

790 LCML Chronic myelogenous leukemia

791 LGG Brain lower grade glioma

792 LIHC Liver hepatocellular carcinoma

793 LUAD Lung adenocarcinoma

794 LUSC Lung squamous cell carcinoma

795 MB Medulloblastoma

796 MESO Mesothelioma 
$797 \quad$ MM Multiple myeloma

798 NB Neuroblastoma

799 OV Ovarian serous cystadenocarcinoma

800 PAAD Pancreatic adenocarcinoma

801 PRAD Prostate adenocarcinoma

802 SCLC Small cell lung cancer

803 SKCM Skin cutaneous melanoma

804 STAD Stomach adenocarcinoma

805 THCA Thyroid carcinoma

806 UCEC Uterine corpus endometrial carcinoma

807 CGP The Cancer Genome Project

808 IC50 Half maximal inhibitory concentration

809 TCGA The Cancer Genome Atlas Program

810 FDA U.S. Food and Drug Administration

811 RTK Receptor tyrosine kinase

812 MEK Mitogen-activated protein kinase kinase 
Figure 1

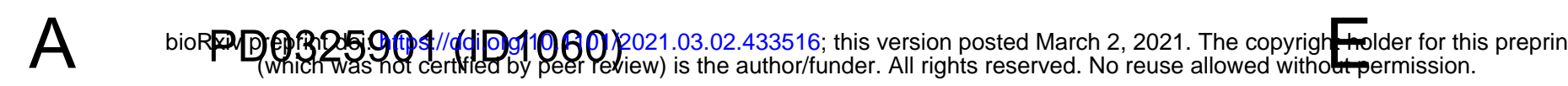

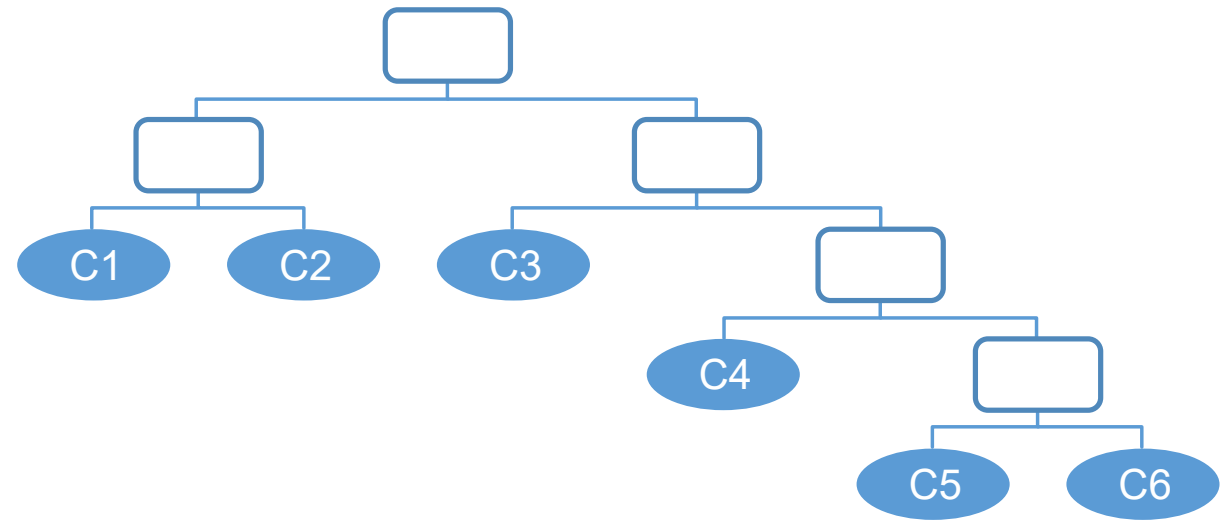

B

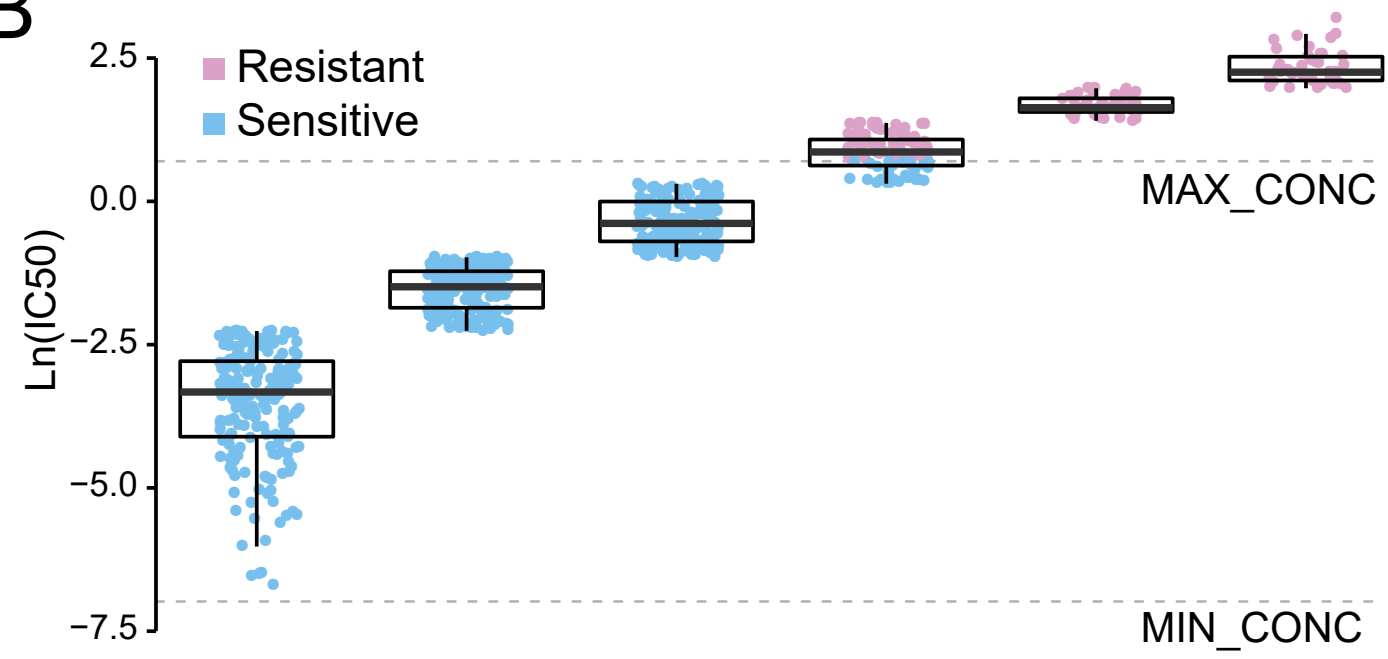

C1

C

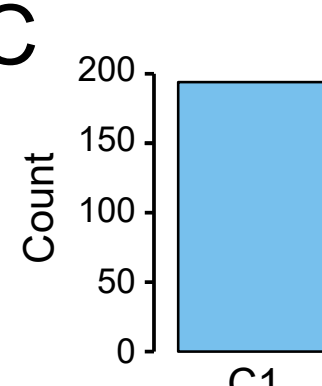

C1
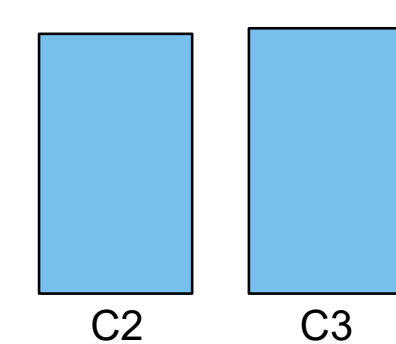

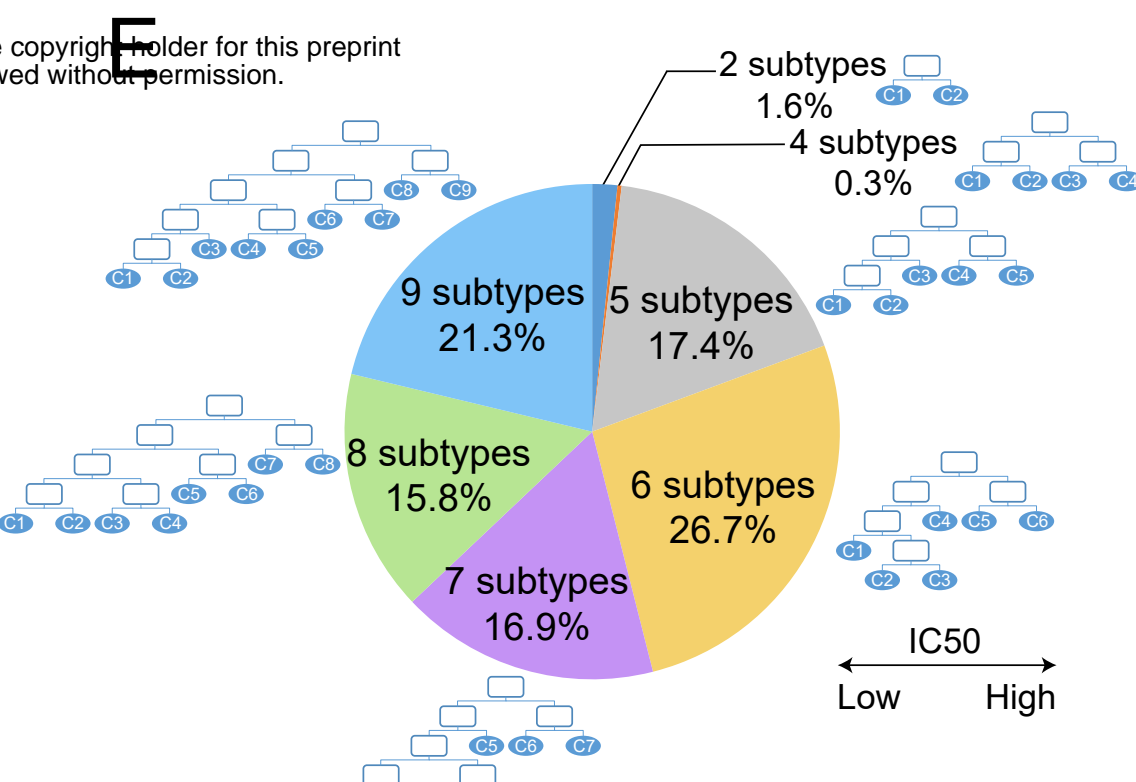

$\mathrm{F}$

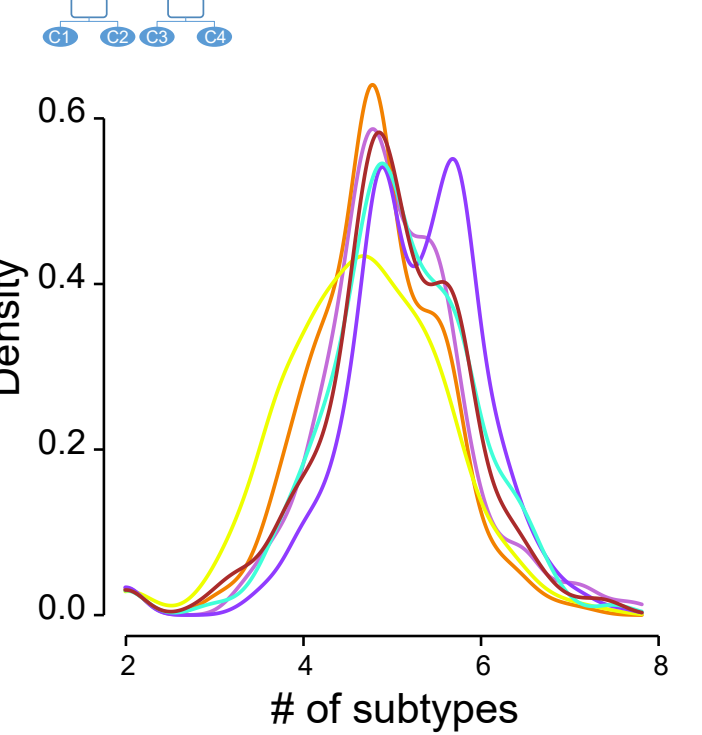

$\square$ Resistant Sensitive

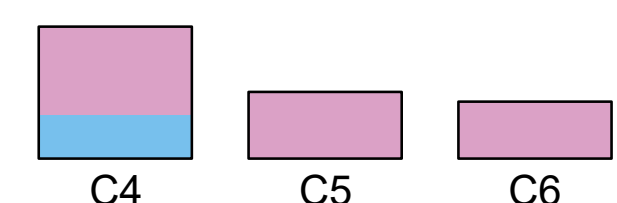

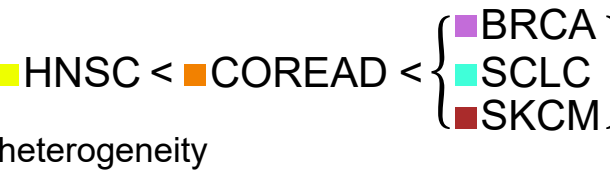

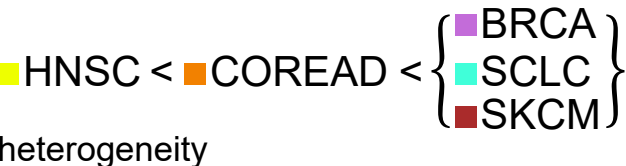

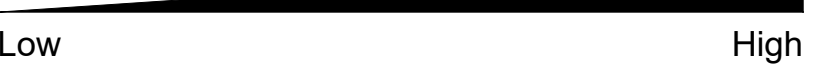

$\mathrm{G}$

D

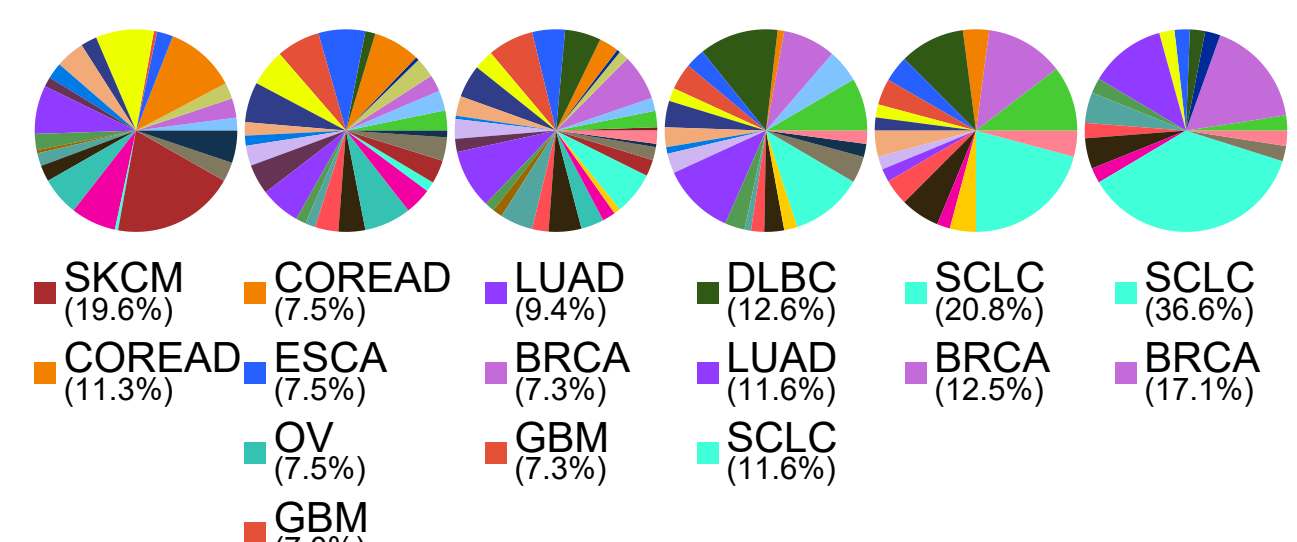

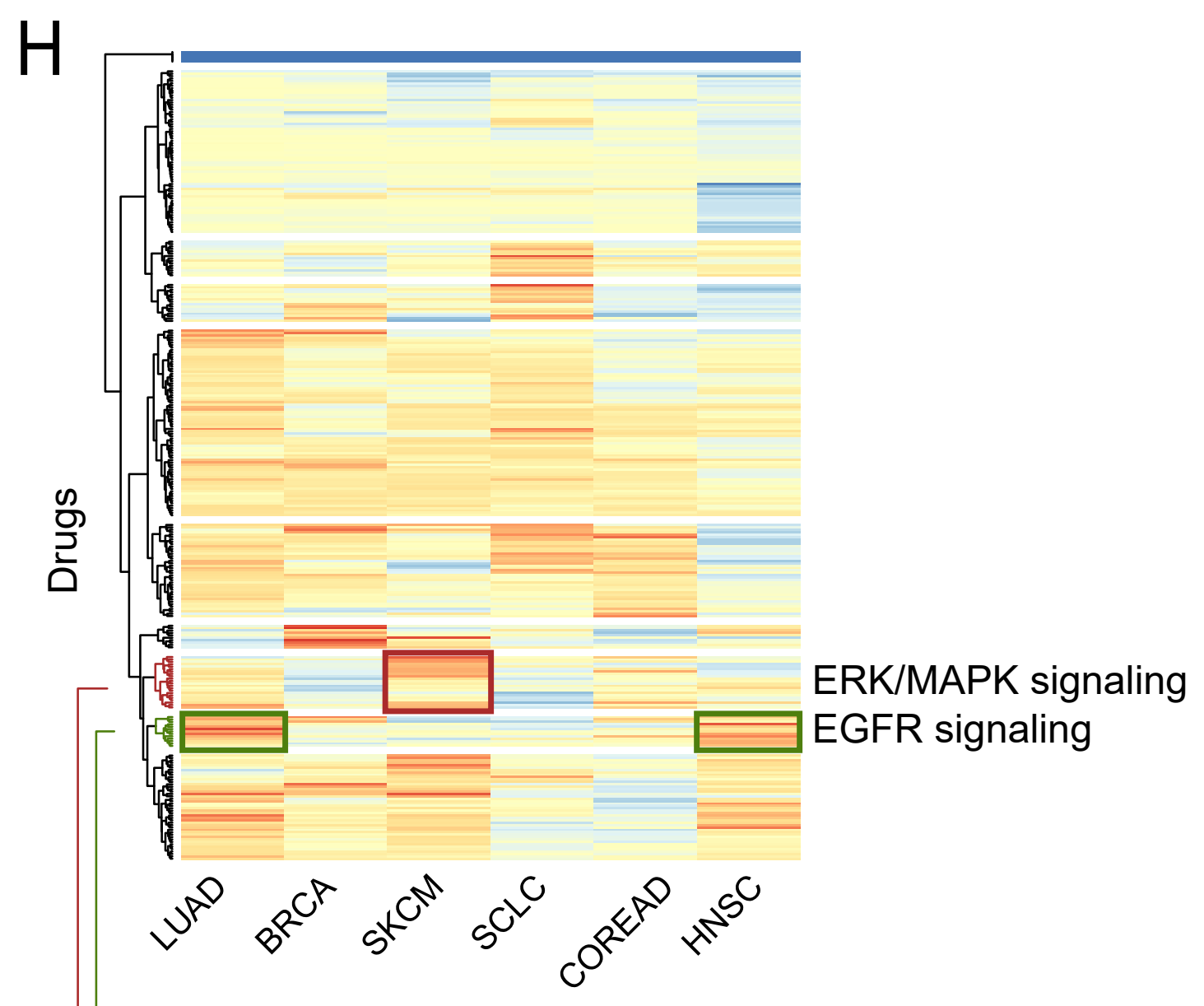

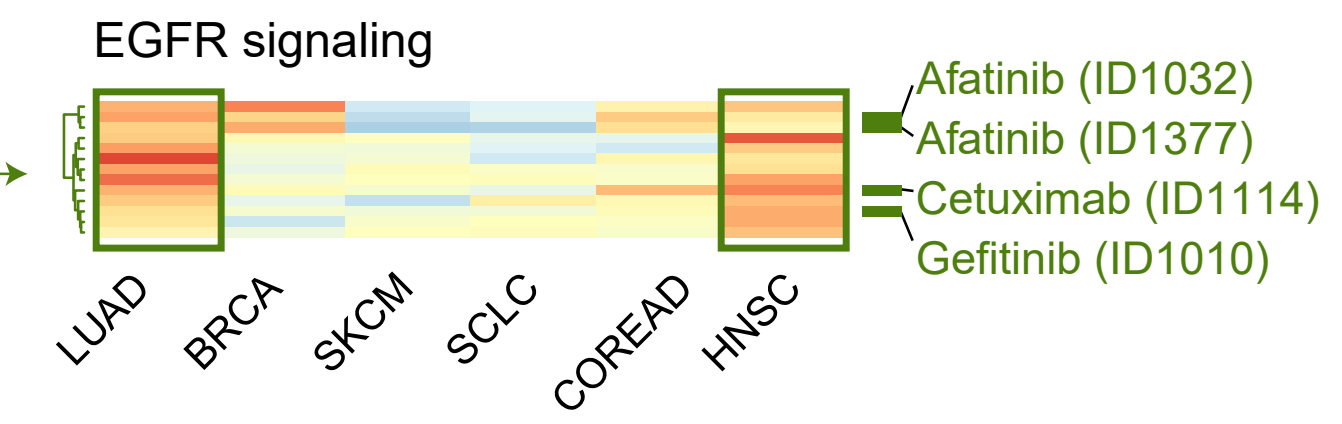
$\left\{\begin{array}{r}\text { SCLC } \\ \square K C M\end{array}\right\}<\square$ LUAD

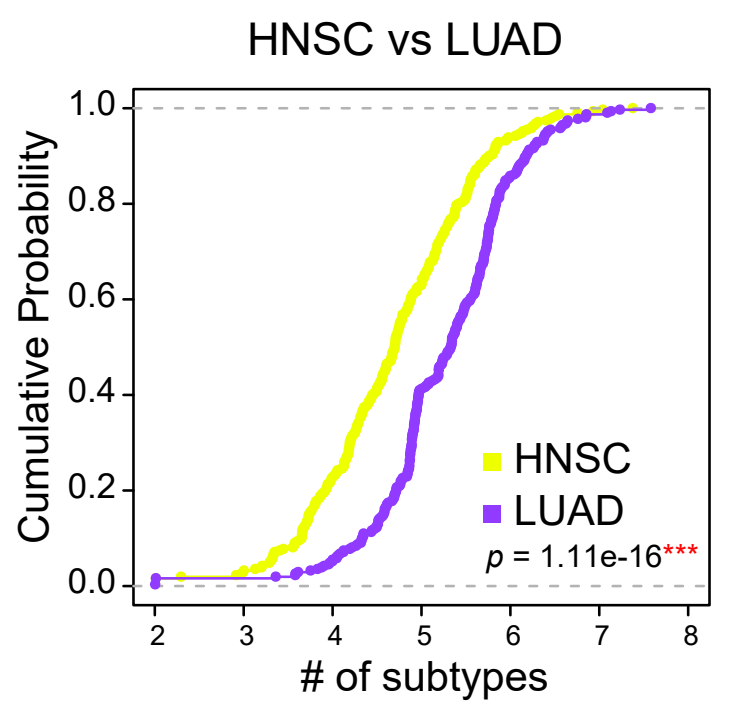

ERK/MAPK signaling $\quad$ SB590885 (ID1061) עVX-11e (ID262) -FR-180204 (ID263) PLX-4720 (ID1371) Selumetinib (ID1498) Dabrafenib (ID1373) Selumetinib (ID1062)
\# of subtypes $\begin{array}{llllllllll}2 & 3 & 4 & 5 & 6 & 7\end{array}$

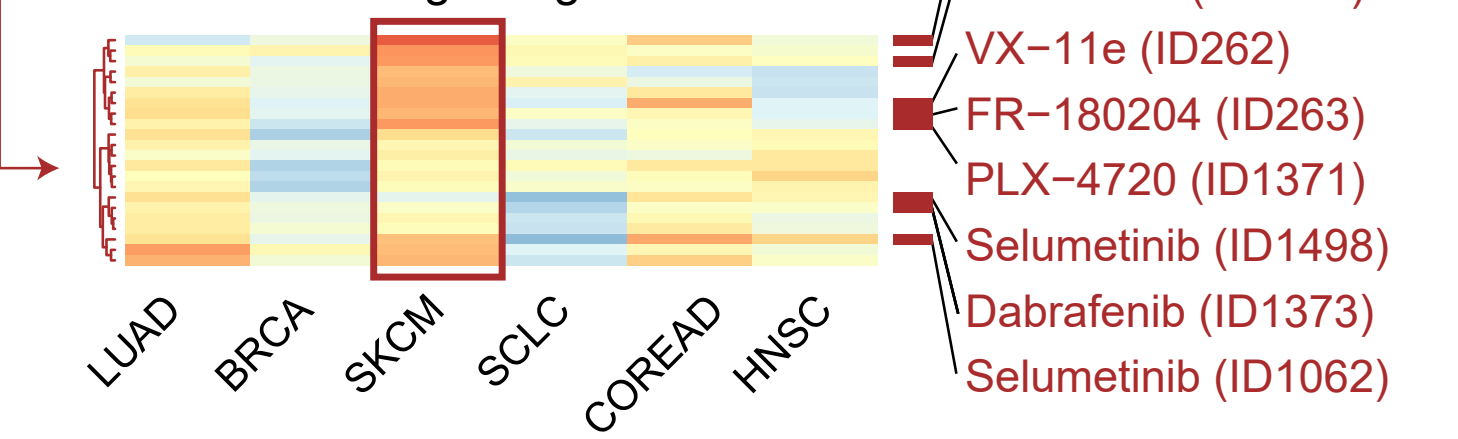
Cancers

$\mathrm{GBM}$
$(7.0 \%)$ 
A

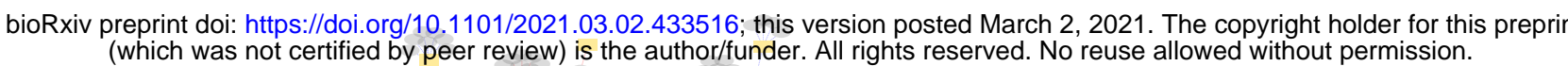

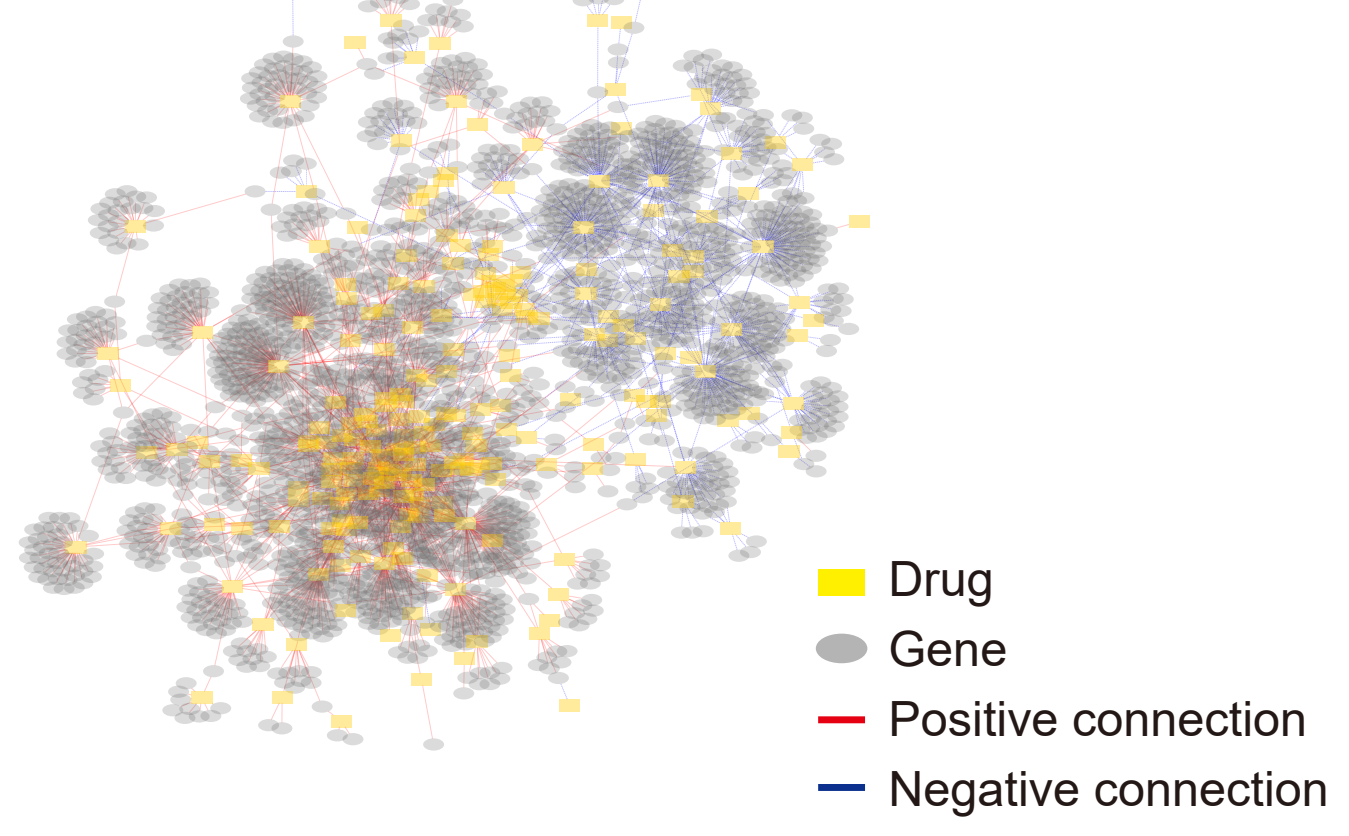

$E$

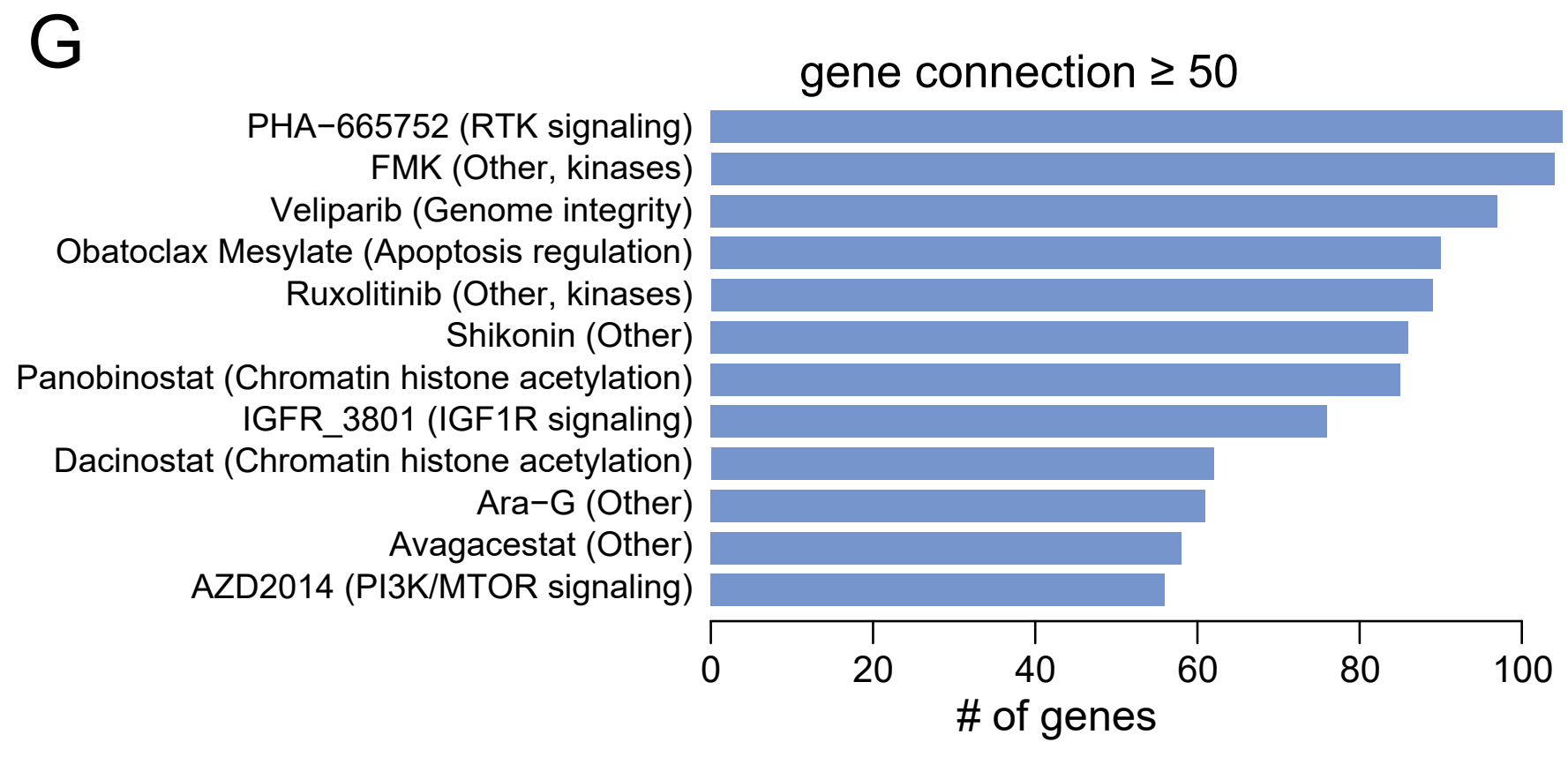

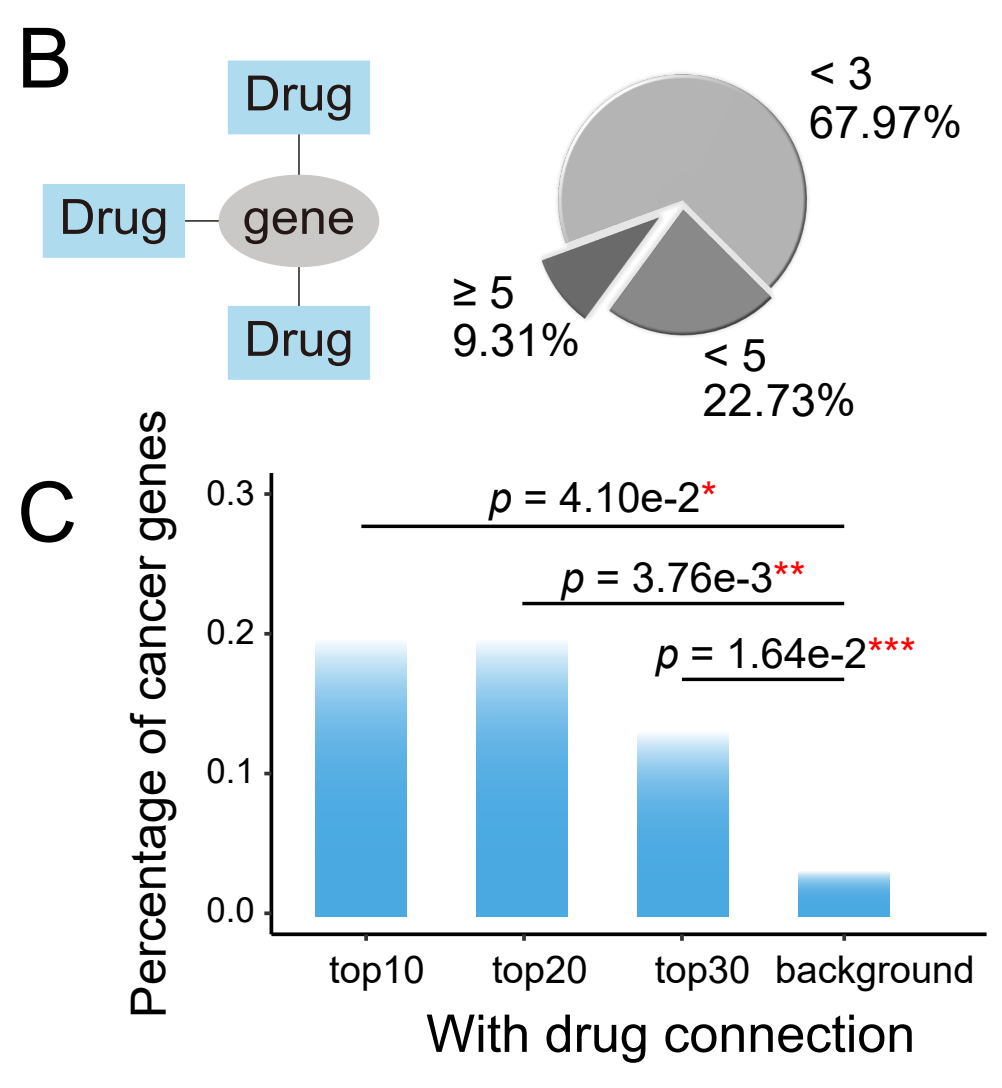

$\mathrm{H}$

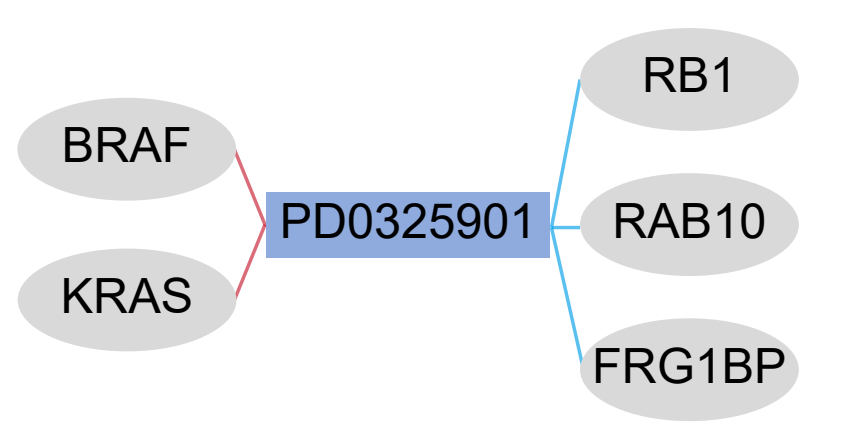

KRAS

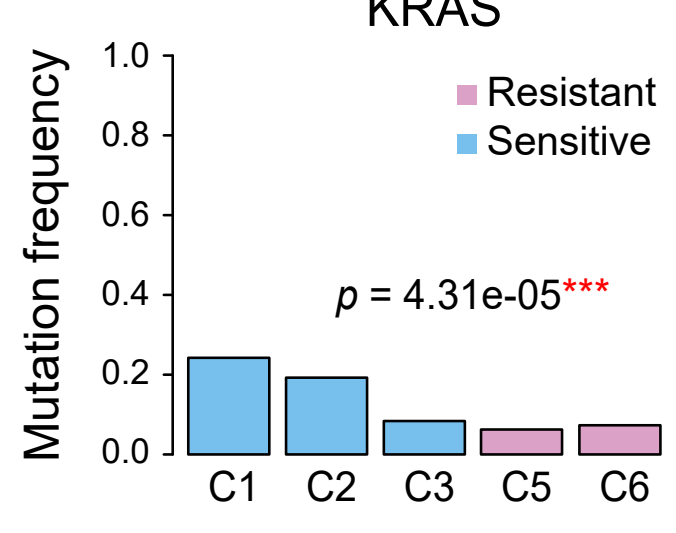

FRG1BP

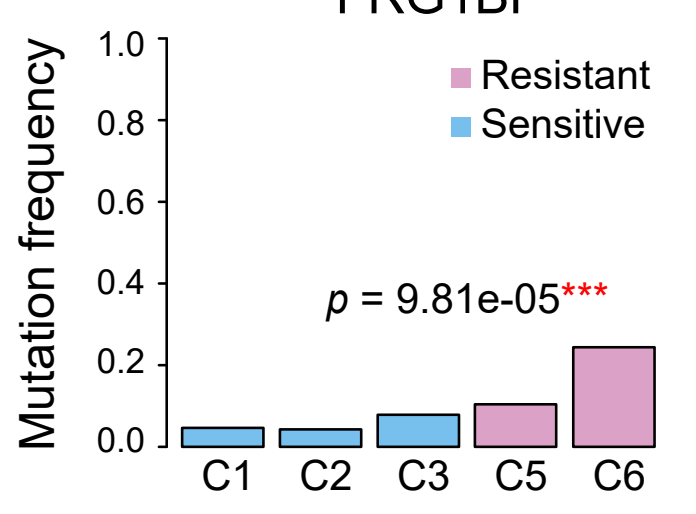

D

\begin{tabular}{cc}
\hline $\begin{array}{c}\text { Cancer } \\
\text { Gene }\end{array}$ & $\begin{array}{c}\text { \# of drug } \\
\text { connection }\end{array}$ \\
\hline BRAF & 13 \\
KRAS & 11 \\
TP53 & 9 \\
RB1 & 7 \\
HSCB-ZNRF3 & 4 \\
VHL & 4 \\
PTEN & 4 \\
MYC & 3 \\
TLX3 & 3 \\
EZH2 & 3 \\
PIK3CA & 3 \\
\hline
\end{tabular}

BRAF

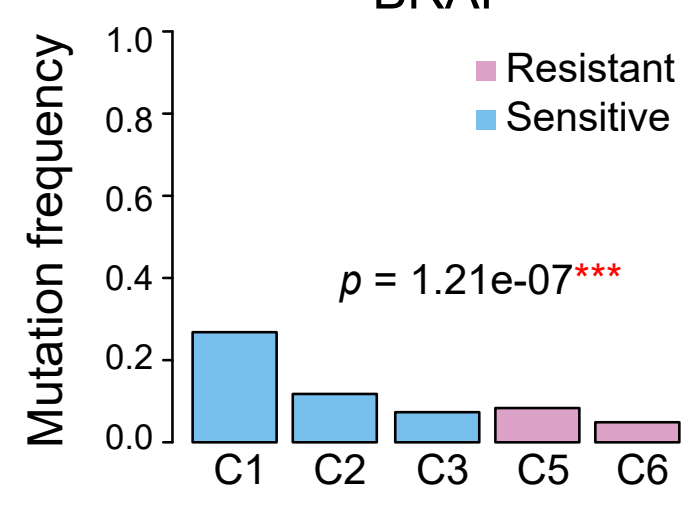

RB1

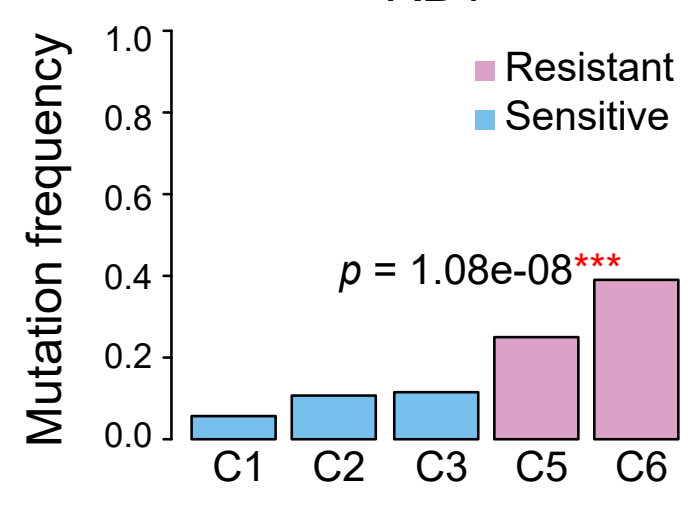

RAB10

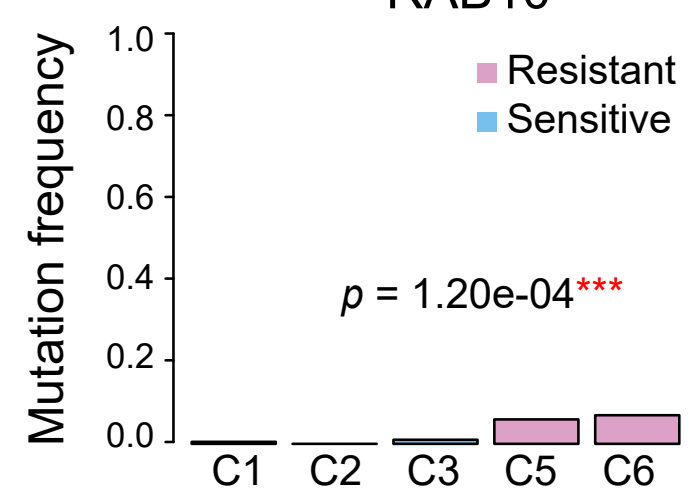


Figure 3

\section{B}

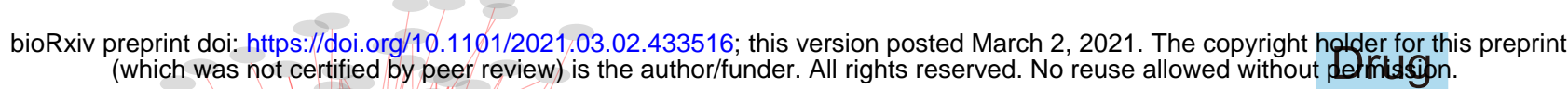

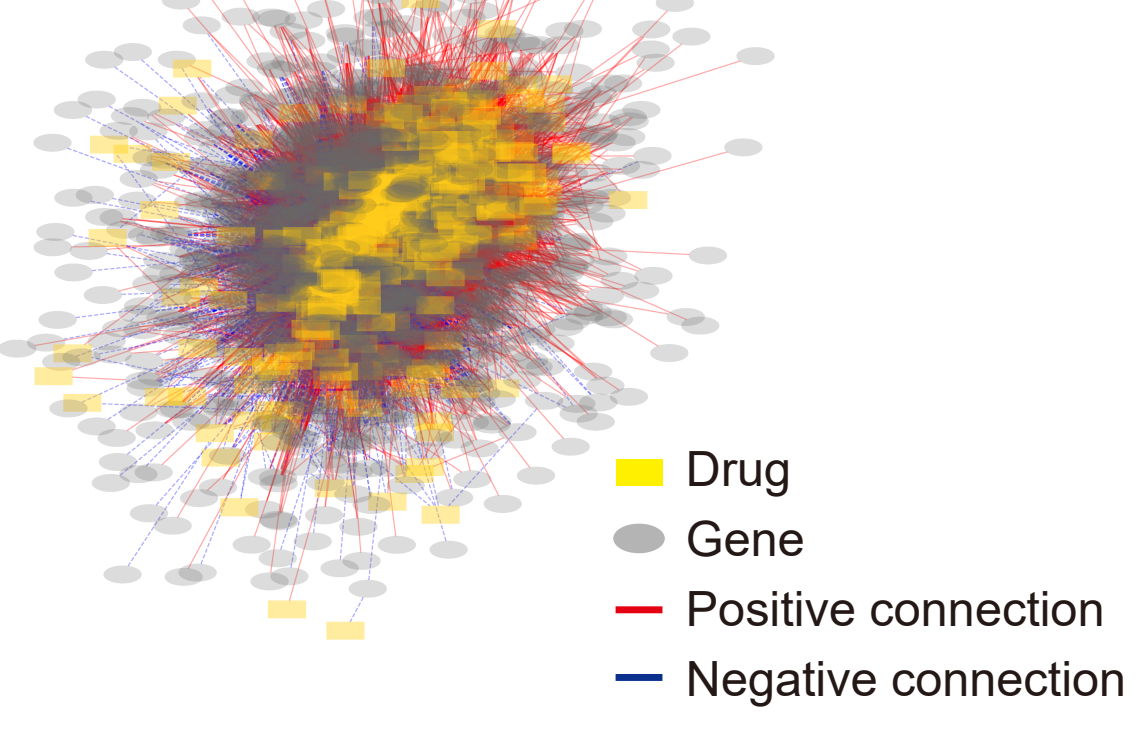

C

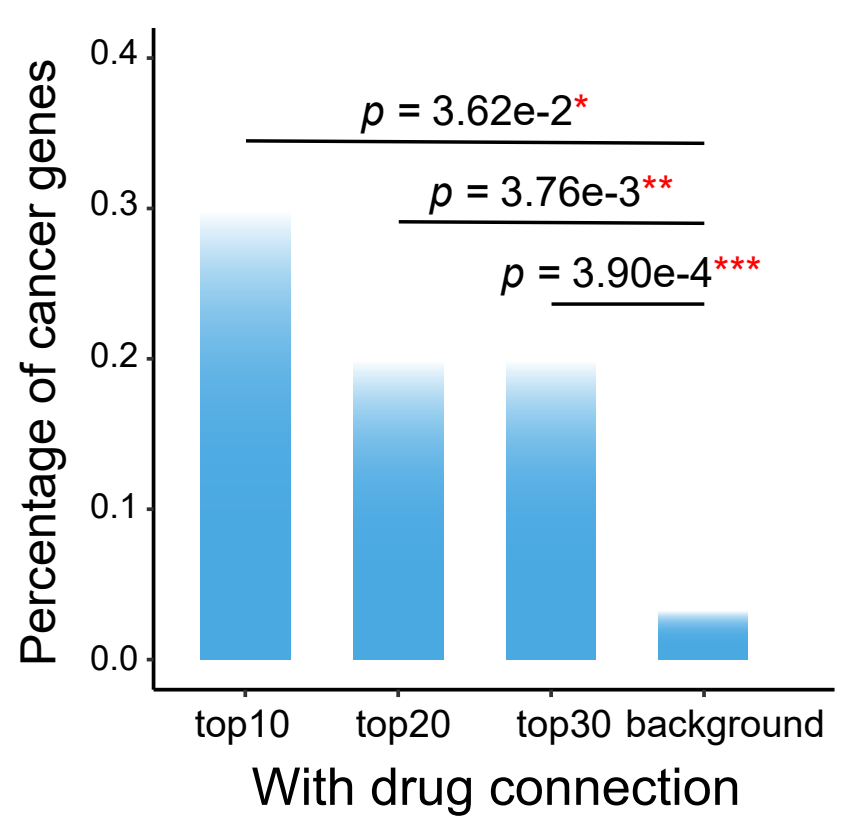

D

\begin{tabular}{cc|cc}
\hline $\begin{array}{c}\text { Cancer } \\
\text { Gene }\end{array}$ & $\begin{array}{c}\text { \# of drug } \\
\text { connection }\end{array}$ & $\begin{array}{c}\text { Cancer } \\
\text { Gene }\end{array}$ & $\begin{array}{c}\text { \# of drug } \\
\text { connection }\end{array}$ \\
\hline IKZF1 & 164 & FANCE & 63 \\
IL21R & 155 & MDM4 & 63 \\
PRKCB & 143 & POLD1 & 60 \\
CD28 & 138 & SPEN & 58 \\
JAK3 & 109 & LEF1 & 57 \\
VAV1 & 103 & SETDB1 & 57 \\
CSF3R & 85 & BAP1 & 56 \\
TERT & 83 & NTRK1 & 56 \\
LCK & 81 & AMAD2 & 56 \\
FBXW7 & 78 & BRIP1 & 55 \\
BCL2 & 75 & MUTYH & 53 \\
WRN & 75 & PPM1D & 53 \\
AMER1 & 74 & ELK4 & 52 \\
CBLC & 74 & NFATC2 & 52 \\
ELL & 74 & BRCA2 & 51 \\
BLM & 70 & CBL & 51 \\
CACNA1D & 70 & ERBB2 & 51 \\
FANCD2 & 70 & MAX & 51 \\
CREB1 & 68 & BARD1 & 50 \\
SMARCA4 & 67 & CCND2 & 50 \\
BRCA1 & 66 & CDK6 & 50 \\
\hline
\end{tabular}

E gene $F$
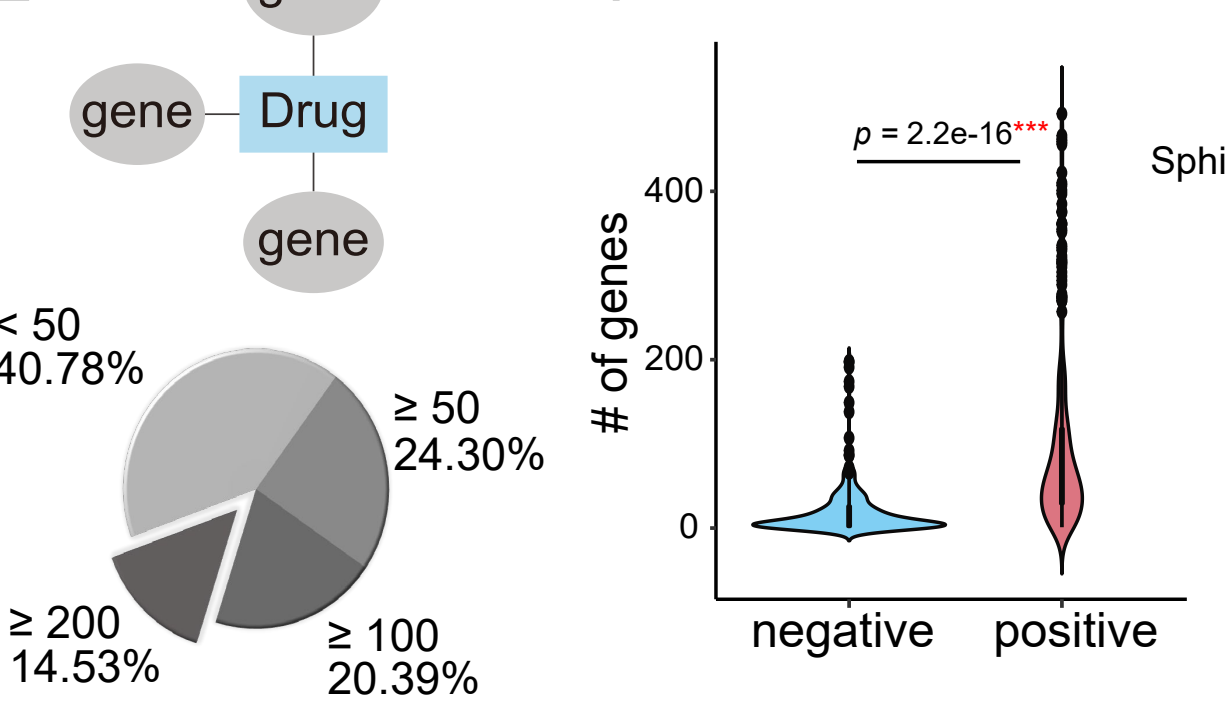

Q P

- Methotrexate -

110

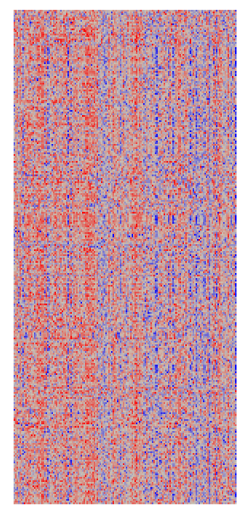

Sensitive

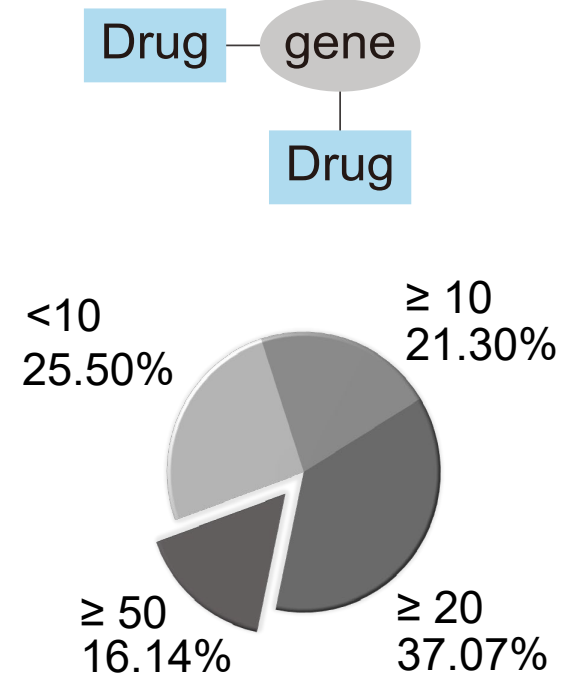

G

EEF2K Inhibitor A-48tentan (Other)

UNC1215 (Chromatin other ETP-45835 (Other, kinases) 1 Inhibitor II (Other, kinases NPK76-II-72-1 (Cell cycle
I-BET-762 (Chromatin othe BMS-345541 (Other, kinases BX-912 (Metabolism) Navitoclax (Apoptosis regulation)
AKT inhibitor VIII (PI3K/MTOR signaling Fedratinib (Other, kinases) 5-Fluorouracil (Othe TPCA-1 (Other, kinases YM201636 (PI3K/MTOR signaling
Methotrexate (DNA replication)
AT7867 (PI3K/MTOR signaling)

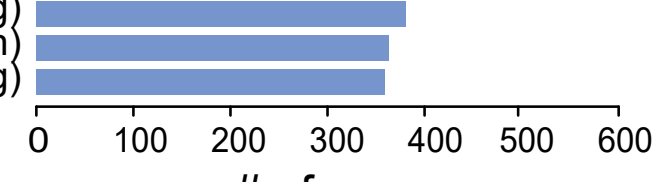

$J$

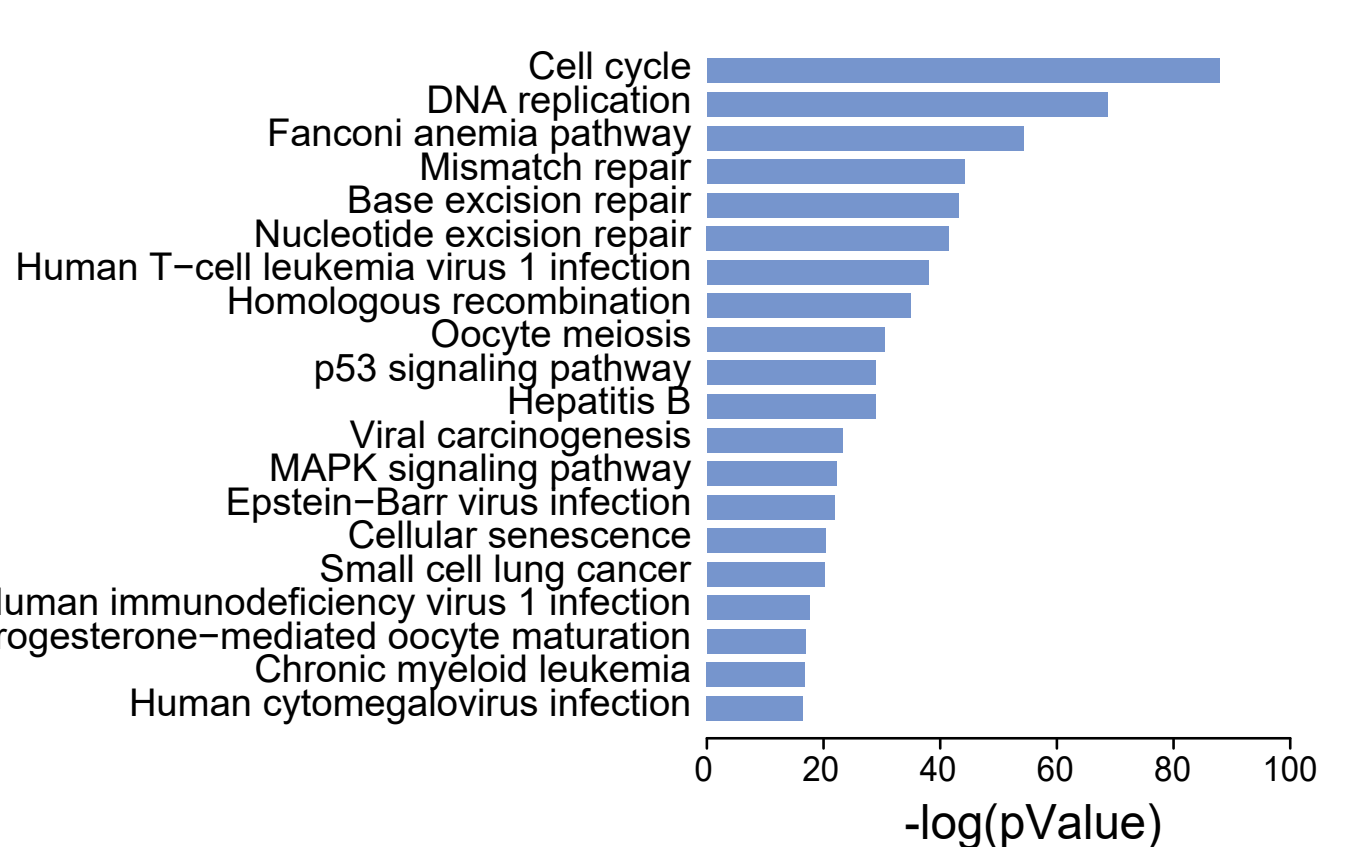

$\mathrm{H}$

Methotrexate (ID1008)
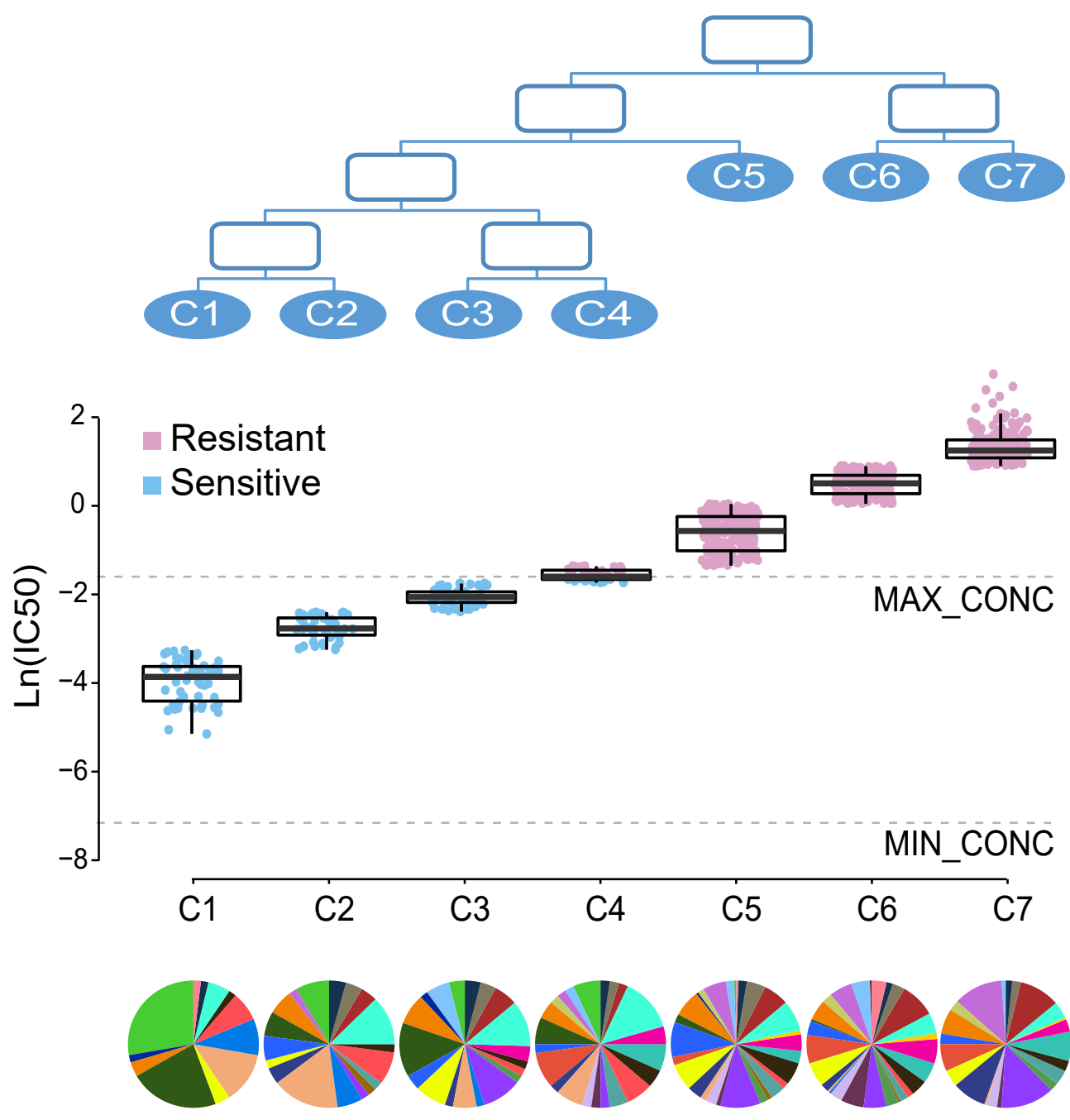

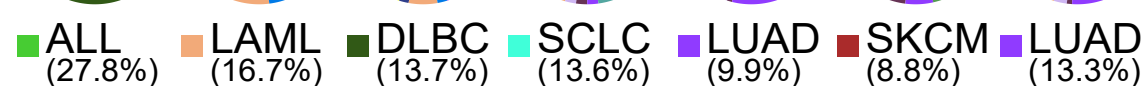

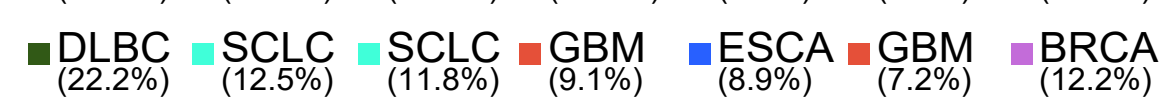


Figure 4

A

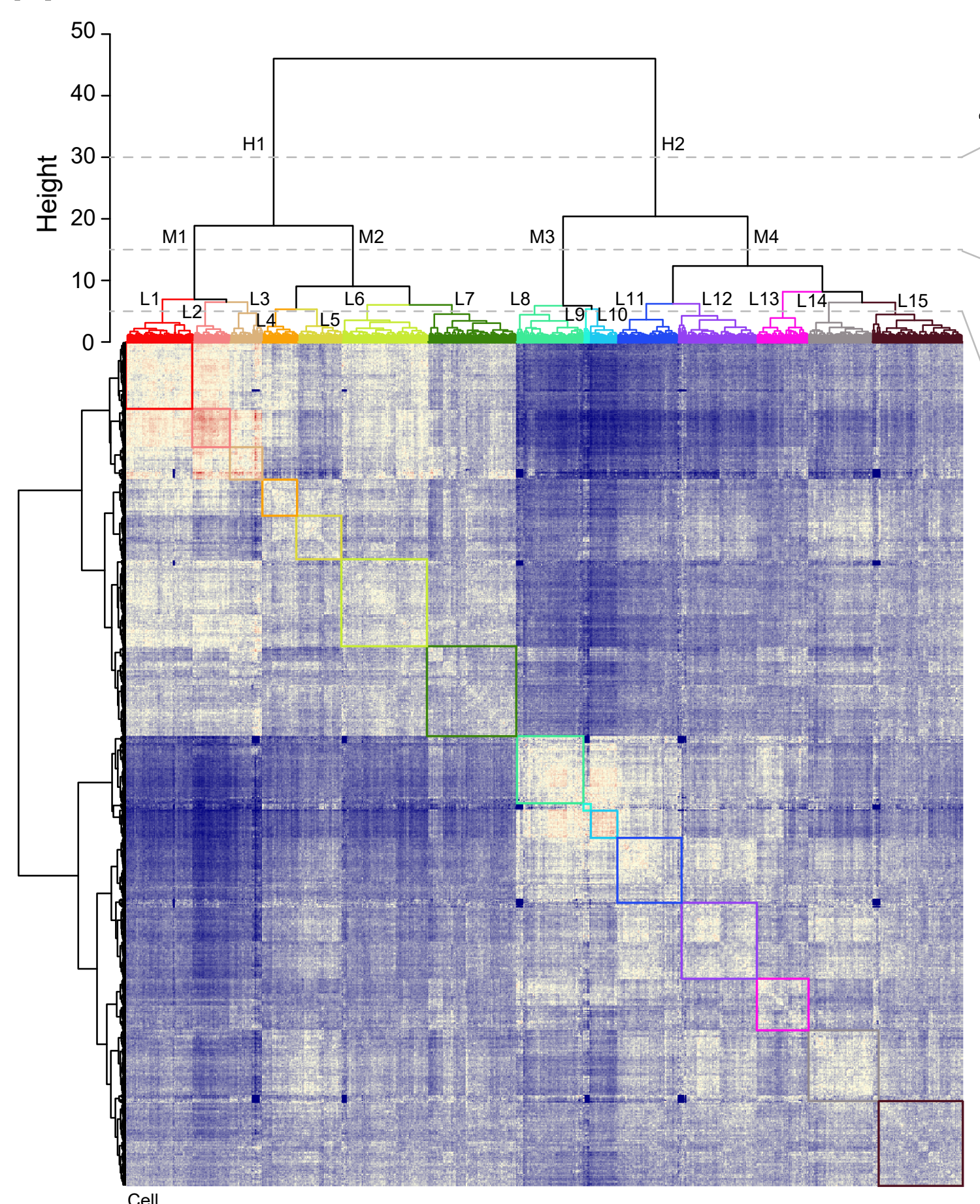

E

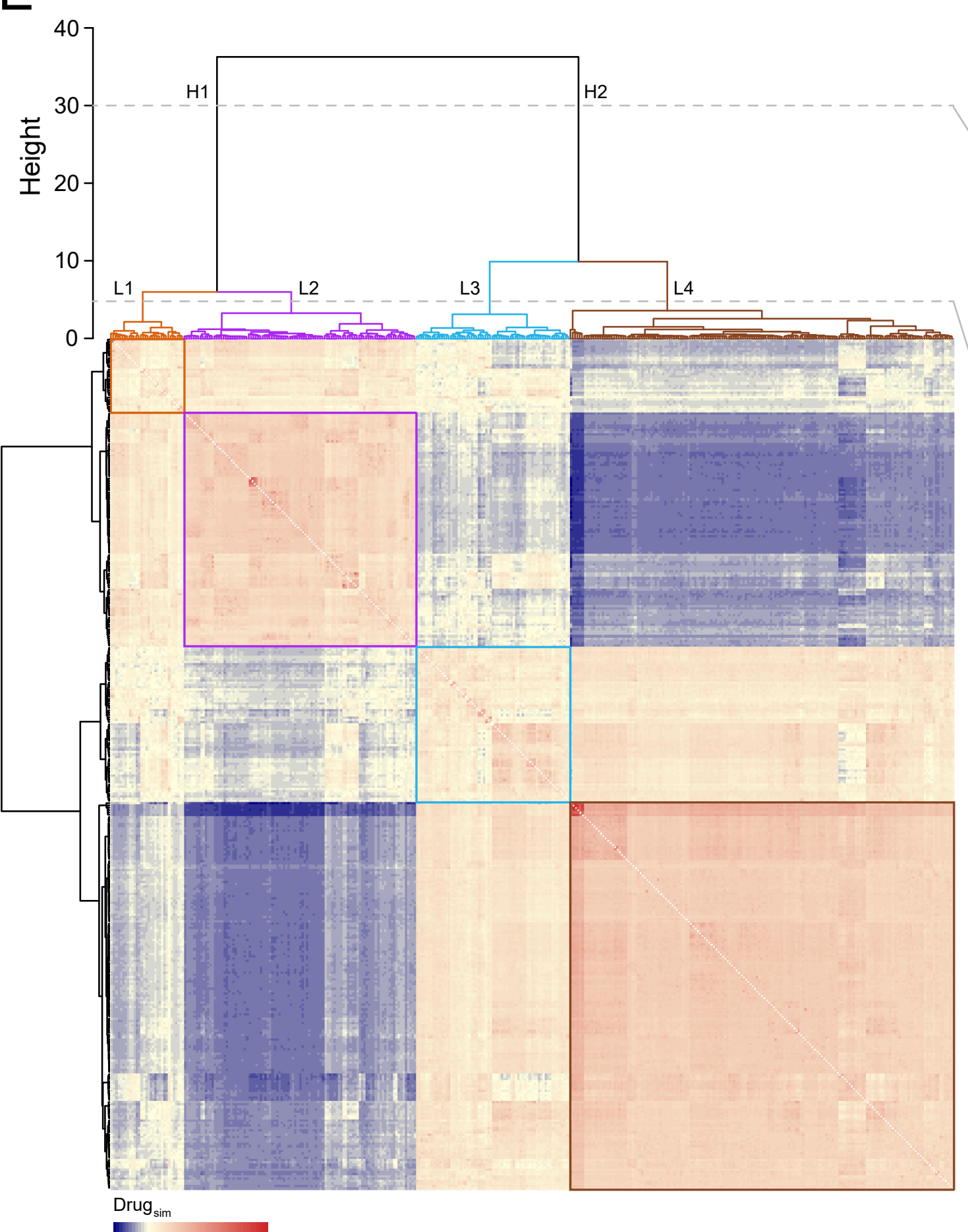

$\mathrm{H}$

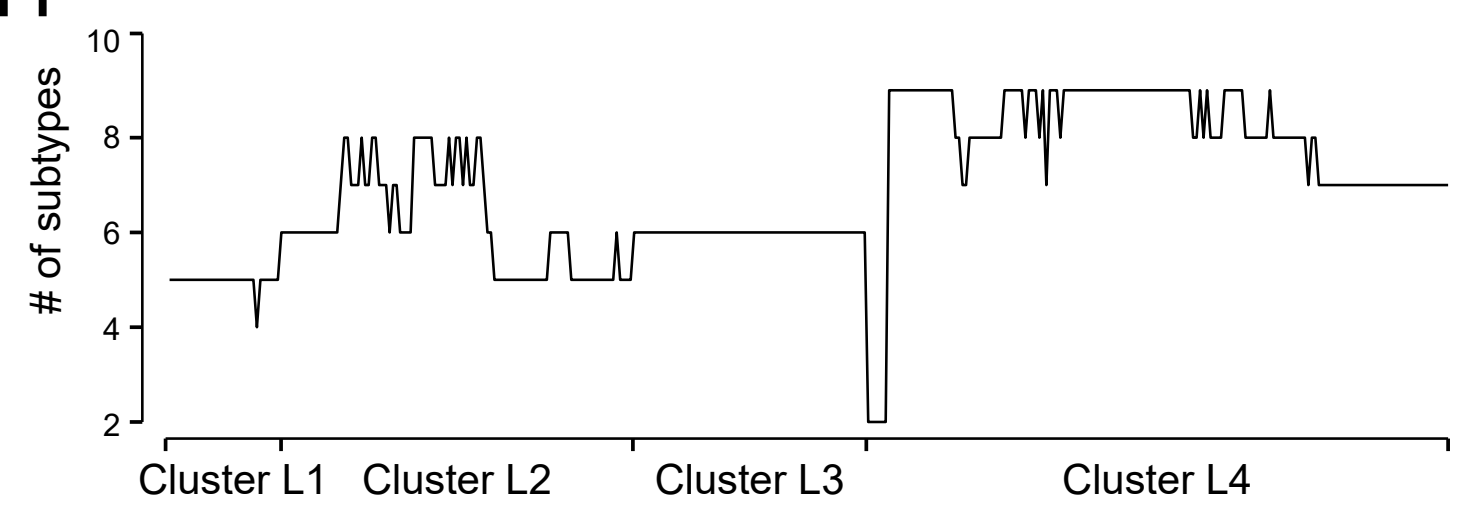

B

Cancer type

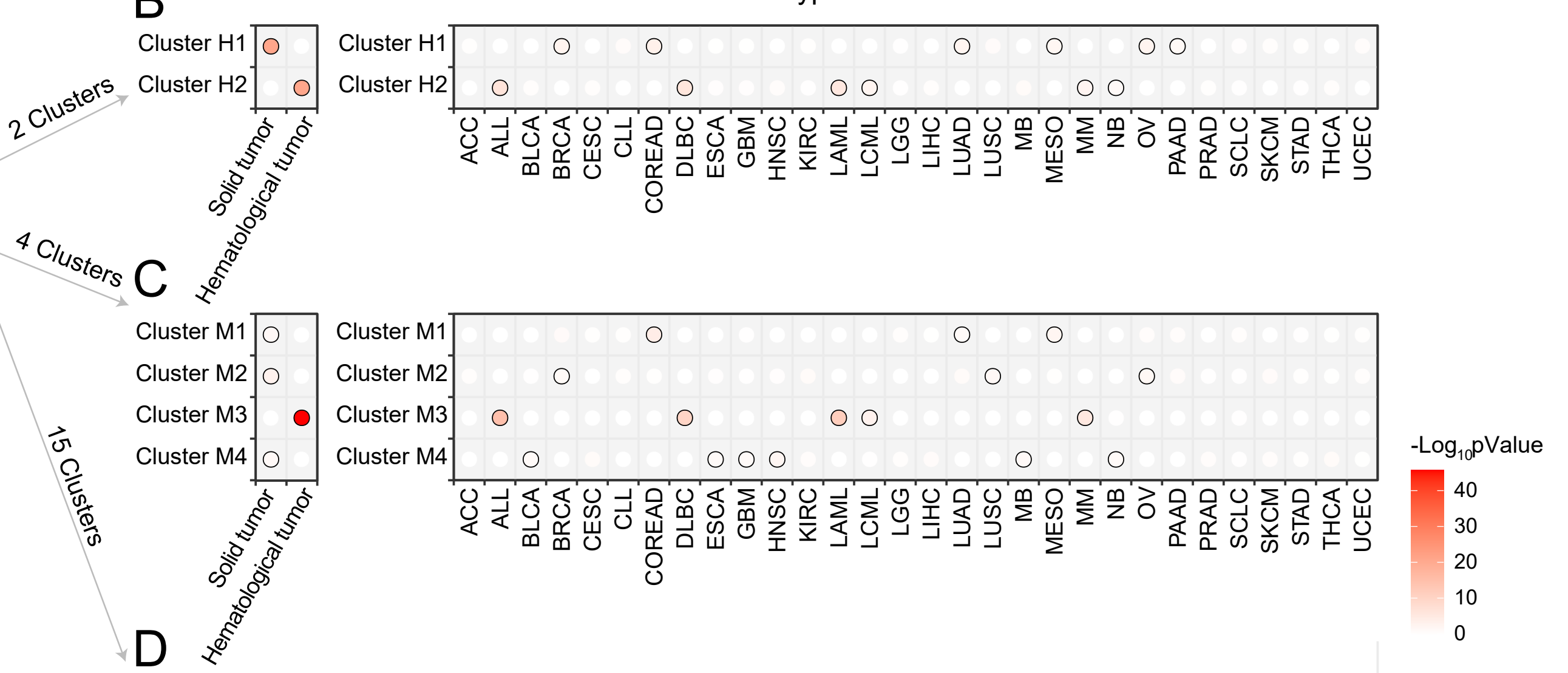

Cluster L1 $\mathrm{O}$

Cluster L2 $O$

Cluster L3

Cluster L4 $\mathrm{O}$

Cluster L5 O

Cluster L6 O

Cluster L7 $\bigcirc$

Cluster L8 $\quad$ Cluster L7

Cluster L9 $\quad$ Cluster L9

Cluster L10 O Cluster L10

Cluster L11 $\bigcirc \quad$ Cluster L11

prequldister L12 O Cluster L12

Cluster L13 $\bigcirc$ Cluster L13

Cluster L14 $\bigcirc \quad$ Cluster L14

Cluster L15 0

Cluster L15
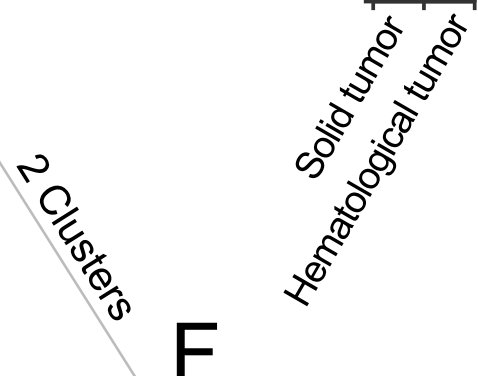

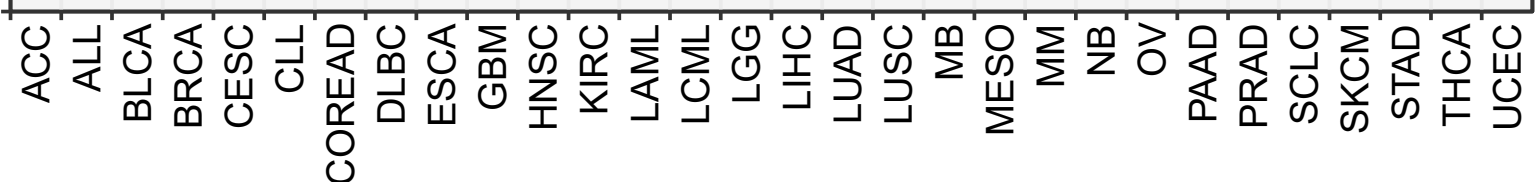

$F$

Pathway
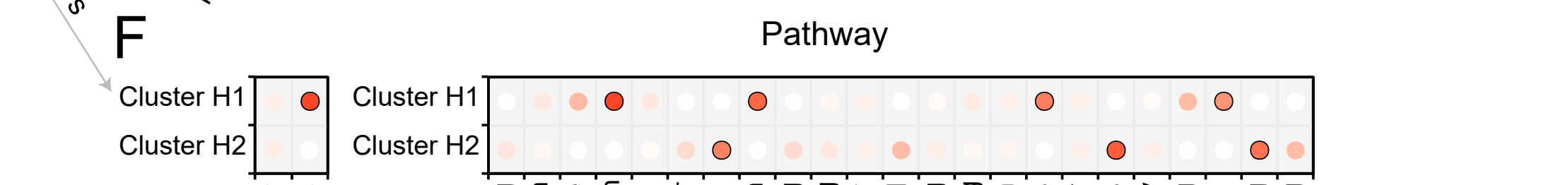

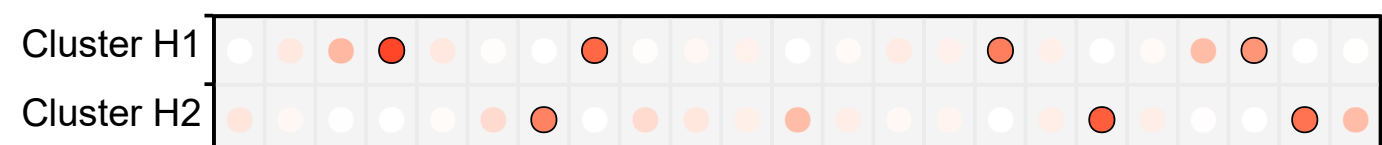

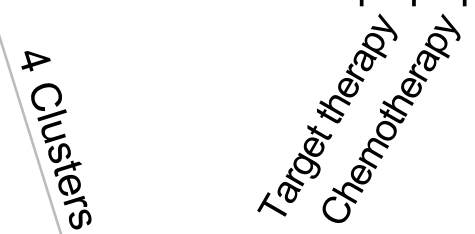

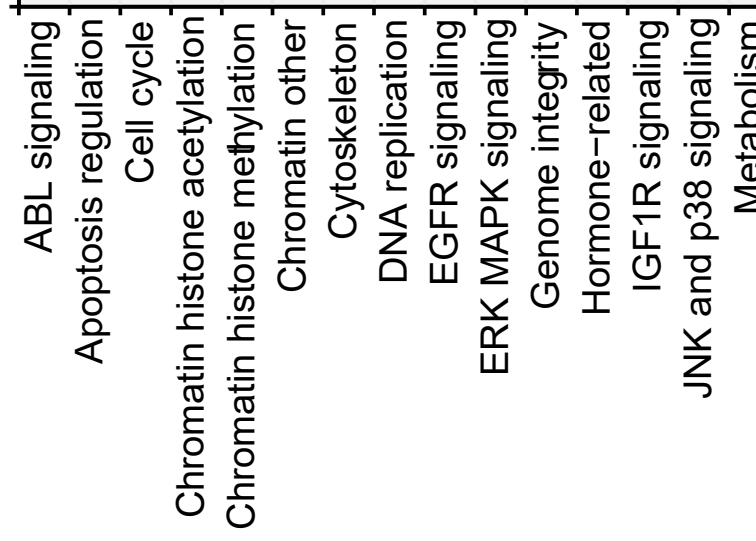

G

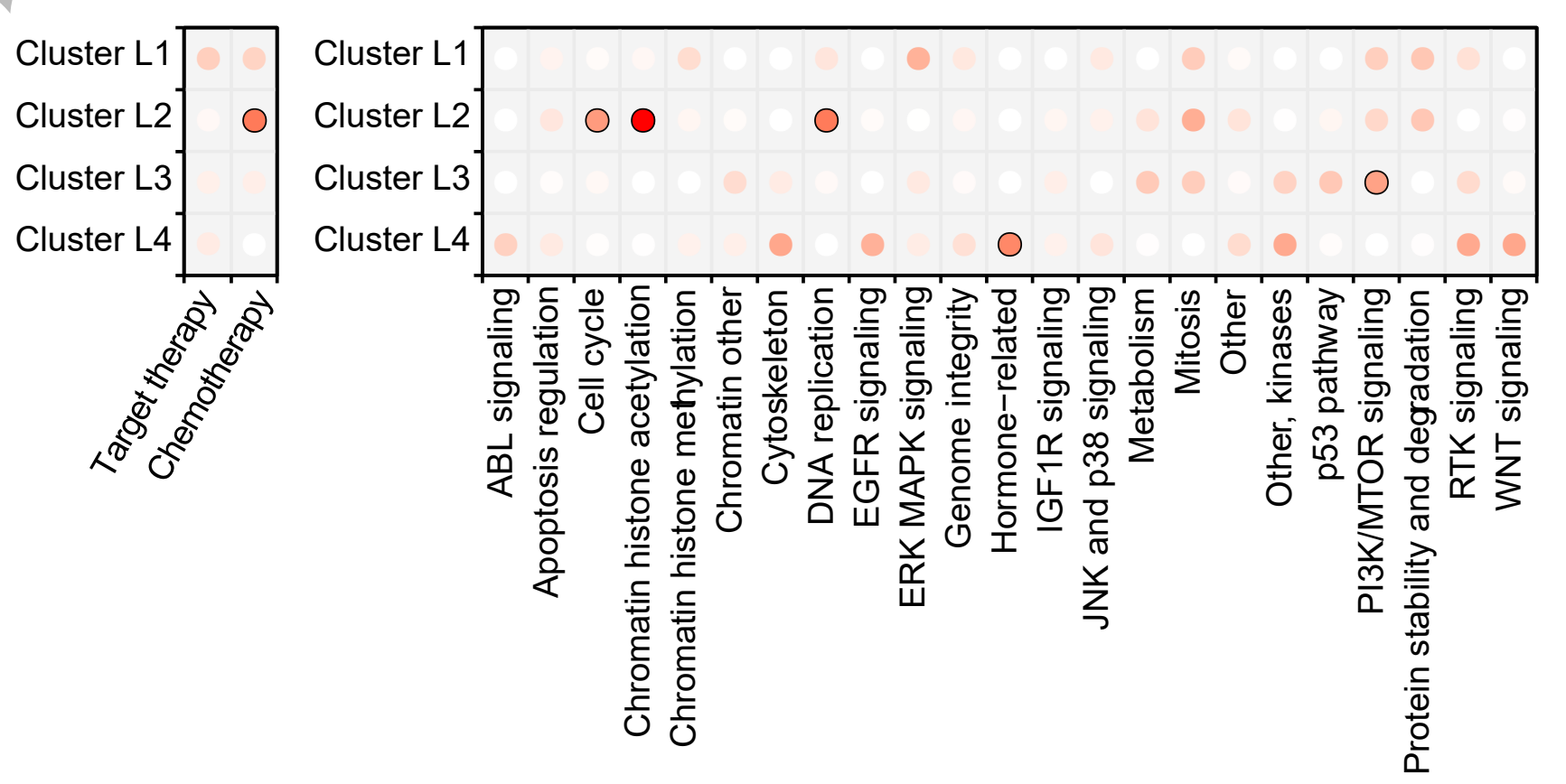

$-\log _{10} p$ Value

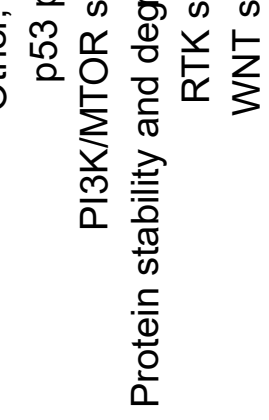

